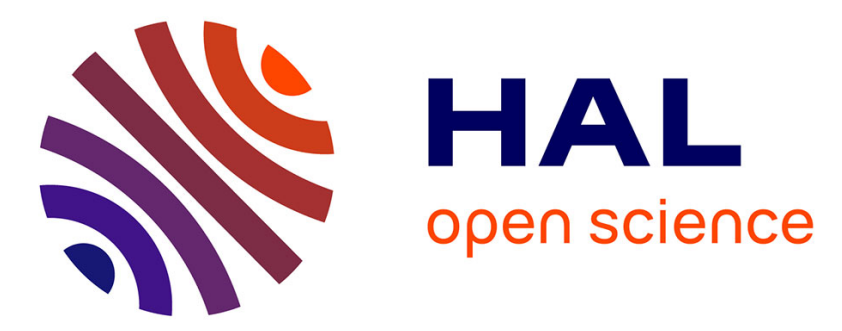

\title{
In-situ Full Field Out of Plane Displacement and Strain Measurements at the Micro-Scale in Single Reinforcement Composites under Transverse Load
}

\author{
I. Tabiai, Damien Texier, P. Bocher, D. Therriault, M. Levesque
}

\section{- To cite this version:}

I. Tabiai, Damien Texier, P. Bocher, D. Therriault, M. Levesque. In-situ Full Field Out of Plane Displacement and Strain Measurements at the Micro-Scale in Single Reinforcement Composites under Transverse Load. Experimental Mechanics, 2020, 60, pp.359-377. 10.1007/s11340-019-00541-z . hal02417490

\author{
HAL Id: hal-02417490 \\ https://imt-mines-albi.hal.science/hal-02417490
}

Submitted on 20 Dec 2019

HAL is a multi-disciplinary open access archive for the deposit and dissemination of scientific research documents, whether they are published or not. The documents may come from teaching and research institutions in France or abroad, or from public or private research centers.
L'archive ouverte pluridisciplinaire HAL, est destinée au dépôt et à la diffusion de documents scientifiques de niveau recherche, publiés ou non, émanant des établissements d'enseignement et de recherche français ou étrangers, des laboratoires publics ou privés. 


\title{
In-situ Full Field Out of Plane Displacement and Strain Measurements at the Micro-Scale in Single Reinforcement Composites under Transverse Load
}

\author{
I. Tabiai ${ }^{1}$ (1) D. Texier ${ }^{2,3} \cdot$ P. Bocher ${ }^{2} \cdot$ D. Therriault ${ }^{1} \cdot$ M. Levesque $^{1}$
}

\begin{abstract}
Micromechanics damage models applied to composites predict stresses and strains in the matrix and fibers as a function of the microstructure, constituting phases mechanical properties and load histories. Material parameters, like interface properties, are identified through inverse methods based on macroscopic stress-strain curves. Predictions are also benchmarked against macroscopic measurements. This situation does not capture local phenomena and hinders the robustness of the indentification/validation process. The purpose of this work is to provide full displacement and strain fields at the scale of a single fibre embedded into a matrix to allow the modelling community to either develop and identify micromechanics damage models or to benchmark their own predictions. Such data is critically lacking in the community. To that end, we have investigated three single fibers having radically different bonding strength with epoxy in addition to a bundle of about a hundred carbon fibers that were used as reinforcements of standard "dogbone" epoxy specimens. A laser scanning confocal microscope (LSCM) is used for micro digital image correlation ( $\mu \mathrm{DIC}$ ) during in-situ quasi-static tests of single-reinforcement dogbone specimens. For all specimens, damage initiated with fiber debonding at the free surface along the tensile direction. The crack then propagates around the interface while slightly growing along the fiber. The interfacial crack is shown to grow faster for couples with weak interfacial bonding. Strong fiber / matrix bonding is shown to stop Mode II transverse interfacial debonding which significantly delays specimen failure. Analysis of the LSCM micrographs with $\mu \mathrm{DIC}$ is used to provide measurements of displacements, strains, and measure depth during each test. The importance of out of plane displacements in interfacial debonding is highlighted. Out of plane displacement is shown to play a role in interfacial crack opening and growth and ought to be considered when studying or modeling damage in FRCs. $\mu$ DIC is shown to be a promising technique to provide a better understanding of the damage mechanisms at the fiber or bundle scales and to determine interfacial toughness of a specific fibre / matrix couple in order to perform accurate damage modeling in FRCs. Displacement, strain, and confidence field results for each pixel from each experiment and at each time step are also provided in an extensive data package for detailed comparison with simulation results.
\end{abstract}

Keywords Digital image correlation · Laser scanning confocal microscopy $\cdot$ Interfacial debonding $\cdot$ Single fiber composite

\section{Introduction}

At the microscopic scale (i.e., $\leqslant 100 \mu \mathrm{m}$, referred to herein as microscale), unidirectionally Fiber Reinforced Composites (FRCs) exhibit a heterogeneous microstructure composed of parallel fibers embedded within a polymeric matrix. At the macroscopic scale, FRCs exhibit anisotropic mechanical properties [1].

M. Levesque

martin.levesque@polymtl.ca

Extended author information available on the last page of the article.
FRCs' microstructure is responsible for their intricate damage mechanisms: interfacial debonding, matrix microcracking, fiber breakage, fiber microbuckling, void growth, among others [1-5].

The World Wide Failure Exercise (WWFE) is an international process that evaluated the predictive capabilities of a number of damage predictive models [6]. The first exercise showed a clear lack of faith in the failure criteria in use during the 1990s-2000s, but also no clear evidence that any criteria provided meaningful failure predictions [6].

In 2013, the second edition of the WWFE evaluated twelve failure theories applied to polymeric matrices reinforced by carbon and glass fibers in a variety of composites, 
geometries and loadings [7]. It focused on triaxial failure criteria. A large scatter and divergence in predictions were observed between simulation results and experiments. The exercise showed that current models can hardly predict every failure mode in 3D [7, 8].

Another conclusion of the second WWFE is that key experimental data is critically lacking to provide meaningful assessment for certain loading cases and damage mechanisms [7]. The third edition of the WWFE started in 2015 and is focused on benchmarking 3D damage models against experimental data. The complete results for this edition are yet to be published. Although various damage prediction models are available, their ability to predict damage for different materials, geometries and loadings remains limited [8].

The field of macro-damage mechanics studies the material's overall behavior with damage modeled as internal variables characterizing the damage growth level, such as crack density growth [1]. Macro-damage mechanics models are often formulated within a thermodynamics framework and macroscopic constitutive equations can often be derived. However, parameters used in macro-damage mechanics are not always directly connected to physical mechanisms [9, 10]. Damage growth level modeling requires damage laws able to describe how, and at which rate, damage features grow through the material [11, 12]. Micro-damage mechanics explicitly models voids and inclusions as part of the microstructure. Constituent material properties are estimated using various methods to obtain averaged quantities [1, 13, 14]. Micro-damage mechanics models are often numerically implemented through computational mechanics. Thanks to the constantly increasing available computing power and the development of damage oriented frameworks and tools, computational mechanics appears to be the mostly adapted field to tackle the complexity of FRCs damage problems. Computational mechanics implementations of micro-damage mechanics can handle a FRC with all its heterogeneities and interfaces, explicitly $[1,11,14-16]$.

Various efforts have been undertaken to conciliate the macro and micro-damage mechanics models. Microdamage mechanics can be used to estimate the material constants needed in the macro-damage mechanics framework. The micro-mechanical computations can be iteratively made while considering increasingly damaged states of the material. Experimental information about the damaged state of a FRC under various loading conditions can thus be used to calibrate such a framework $[1,17,18]$.

When transversely loaded with respect to the fibers' direction, damage in unidirectional FRCs initiates through fiber / matrix interfacial debonding at a free surface. This damage mechanism has been reported to be the most critical under transverse loading [1, 19, 20]. A variety of tests have been used to characterize the fiber/matrix interface: the pullout, the push-out, the three fiber and the microbond tests [1, 21]. These methods experimentally measure the averaged fiber / matrix interfacial shear strength (IFSS). The IFSS measures the Mode II intefacial fracture toughness [11]. However, interfacial debonding is a mechanism which has been reported to occur under mixed-mode conditions [1, 11, 22]. Thus, the IFSS might not be sufficient to completely characterize and validate interfacial debonding models for FRCs subjected to macroscopically transverse loading $[11,19]$.

Damage modeling related to interfacial damage is still an open problem being studied [20, 23]. Damage for a single fiber composite under transverse loading was investigated by Martyniuk et al. [19]. Through X-Ray Computed Micro-Tomography $(\mu \mathrm{CT})$, the authors demonstrated how tunneling (i.e., interfacial debonding along a fiber's axis) directly impacted the interfacial debonding at the specimen's free surface for an E-glass / epoxy single fiber composite. The observations documented and validated damage mechanisms and predictions for theories for which experimental data was lacking. No local or full field displacement measurements were done in that work. Accurate knowledge of the fiber / matrix interface, and its behavior, during damage growth is required to model an experimental case similar to that studied by Martyniuk et al. [19].

Most damage observations of single fiber composite and FRCs found in the literature were performed using Scanning Electron Microscopes (SEM) $[24,25]$ or $\mu \mathrm{CT}[15,19]$. Xrays have been reported to damage epoxy specimens during observation and might change material behavior at the free surface [19]. In addition, out of plane measurements can hardly be obtained with a single detector under a SEM. The Laser Scanning Confocal Microscope (LSCM) technique consists in capturing images at different regular depth intervals, blocking out of focus light at each depth. The method thus enables reconstructing three dimensional structures and out of plane measurements.

Digital Image Correlation (DIC) is an optical method for computing 2D and 3D displacement fields based on a crosscorrelation between an initial and deformed images. The method requires a random grey level pattern that can either exist naturally on the specimen or be produced artificially (e.g., speckle pattern created with paint droplets).

Micro digital image correlation $(\mu \mathrm{DIC})$ has already been applied to FRCs to measure displacements and strains at the fiber scale [24, 25]. Canal et al. first used $\mu$ DIC to investigate strain fields in a unidirectional E-glass / epoxy FRC under transverse compression. Submicron alumina particles were dispersed on the specimen's surface as a speckle 
pattern. A SEM was used to capture images. The study showed the potential for applying $\mu \mathrm{DIC}$ to FRCs' surface displacement measurements, although strain concentrations around fiber bundles were reported as difficult to identify [24]. Mehdikhani et al. pursued this line of work a few years later by performing a similar experiment on an Eglass / epoxy specimen loaded using a three-point bending setup [25]. The authors improved the method used to deposit submicron particles, which made detection of strain concentrations due to fiber bundles possible. However, the authors reported difficulties measuring displacements in the vicinity of isolated fibers due to the speckle pattern's properties and limitations of the subset based DIC method [25].

No damage, and specifically no interfacial decohesion, was observed during these experiments [24, 25]. Nevertheless, the matrix' out of plane behavior around a single fiber was not investigated while damage is growing by Martyniuk et al. [19]. Other authors have compared local strain fields measured by DIC with numerically predicted fields in FRCs submitted to transverse loads [15, 16, 22, 24, 25].

To the best of our knowledge, out of plane measurements and out of plane interfacial cracks have not been quantitatively studied yet, for single fibers or bundles during damage initiation and growth.

DIC results can be used to benchmark micro-mechanical damage models through comparisons with results from numerical simulations. Cohesive zone modeling [11] or X-FEM [12] are often used to model damage and crack growth in materials. Accurate measurements related to damage features could be used to better model damage for both methods. X-FEM enriches Finite Element (FE) shape functions in discontinuities areas and experimental data about crack growth could be used for that purpose. Moreover, the use of cohesive elements requires the a priori knowledge of the crack path, which can be obtained from experiments. Cohesive elements within a FEM mesh obey a cohesive traction law which allows specific elements to deform while transmitting stresses until a certain stressstrain threshold is reached. Experimental data revealing the behavior of different interfaces and materials during damage initiation could be used to calibrate such laws. Richefeu et al. showed how DIC results could be used to derive cohesive zone laws from full field experimental measurements from metallic materials [26]. The team proposed a methodology to derive a cohesive law out of full field measurements of a Mode I crack. A different team, Sakanashi et al., used DIC to analyze a particulate reinforced composite during a wedge splitting test. The main goal of the study was to model fracture using cohesive zone modelling for the studied composite. The team relied on DIC to detect crack areas within the observed surface, calculate the crack tip opening angle and crack tip opening displacement. A traction separation law was then derived based on the crack tip opening displacement, providing a cohesive zone modelling for the studied particulate reinforced composite [27].

This paper investigates the microscopic damage behavior for three different single fibers, and a bundle of carbon fibers, embedded into the same epoxy matrix under quasistatic transverse loading. The fiber materials used were selected for their different bonding strength with the epoxy matrix. In-situ laser scanning confocal microscopy (LSCM) tensile testing was used to document the full displacement and strain fields obtained through DIC analysis in addition to out of plane measurements. More specifically, the purpose of this study is :

- to provide accurate full field experimental measurements of interfacial debonding and damage initiation in a fiber's vicinity

- to provide a better understanding of damage initiation and growth in a FRC quantitatively

- to provide full and complete experimental datasets that can be further compared with simulations to improve or benchmark micromechanical damage modelling

The full displacement and strain measurements, out of plane measurements, load cell force and cross-head displacement are provided as an additional dataset.

We wish to emphasize at this stage that the main motivation for our paper stems from the fact that full experimental strain fields, at the scale of the fibers and matrix, during damage in FRCs are not currently and openly available. As demonstrated in this paper, obtaining such information, with high fidelity, requires considerable efforts. We believe that the data set we provide with this paper will help the modelling community to not only qualitatively assess their models relevance, but will also provide means to validate quantitatively their predictions, which is currently critically lacking. We hope that such benchmark will provide the required confidence to deliver more accurate damage predictions models to better predict composites damage in real engineering applications.

Section "Materials" introduces the materials and samples used. Section "Methods" presents the in-situ microtensile test setup used, along with the image acquisition apparatus and DIC settings. Section "Results" presents the full strain field measurements for each specimen, along with the local strain measurements around reinforcements and the out of plane displacement measurements. Finally, Section "Discussion" presents the differences in damage mechanisms, strain fields and out of plane displacements between strongly bonded and weakly bonded specimens, the limitations of this work and additional elements deemed relevant for future comparison. 


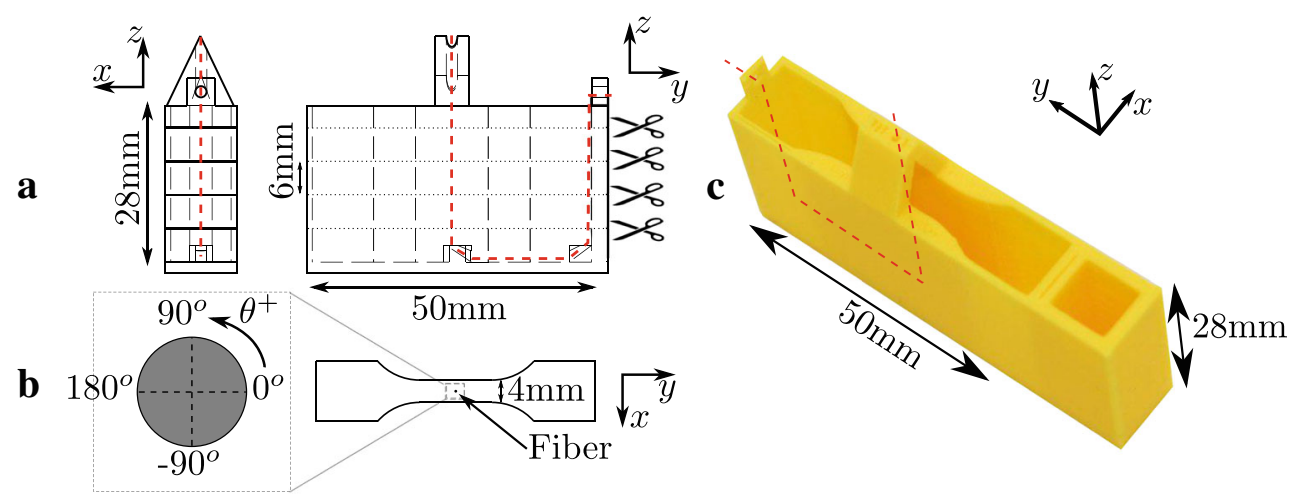

Fig. 1 (a) Computer aided design of the 3D printed mold. The red dashed line shows how the reinforcement was held in the mold. The mold was cut using an abrasive-waterjet cutting machine along the scissor lines to obtain $6 \mathrm{~mm}$ thick individual dogbone specimens containing a single reinforcement transversely to the tensile direction. (b) Modified ASTM D638 Type V specimen with an embedded transverse fiber. Load is applied along the $y$ direction

\section{Materials}

\section{Specimen Fabrication}

A mold was designed to manufacture three types of single fiber composites and a composite reinforced by a bundle of fibers. Figure 1(a) schematically shows the mold's front and right side views. The specimen's geometry was adapted from ASTM D638 Type V standard geometry to accomodate the micro-tensile machine's geometry [28]. The molds were 3D-printed using a fused deposition modeling printer. The molds contained a path to maintain the reinforcement in place during matrix curing. Each mold was first coated with Frekote 700-NC releasing agent. The reinforcement was then inserted into the mold and both its ends were attached on the mold's outside surface.

The red dashed line depicted in Fig. 1(a) shows the reinforcement's position in the mold.

Ten parts by mass of Epon ${ }^{\mathrm{TM}} 862$ resin was mixed with four parts of Epikure ${ }^{\mathrm{TM}} 3274$ hardener to manufacture the matrix. The mixture was first degassed in a large plate to accelerate degassing for $\approx 30$ minutes, poured into the mold, degassed again and then left to cure for $24 \mathrm{~h}$ at room temperature. Once cured, each mold was cut following the scissor lines shown in Fig. 1(a) using an abrasive-waterjet cutting machine. This process was selected to prevent damage induced by traditional material cutting techniques [29]. Each slice obtained was manually extracted, providing a "dogbone" specimen similar to that shown in Fig. 1(b). Every specimen was then polished with silicon-carbide abrasive papers grit P320, P640, P800, P1200, P2000 and P4000, consecutively for a smooth finish.

\section{Material Properties}

Four pure epoxy ASTM D638 Type I specimens were manufactured and mechanically tested according to ASTM
D638 standard. The specimens were tested using a MTS Insight $₫$ electromechanical testing machine equipped with a $25 \mathrm{kN}$ load cell. Displacements were recorded using a 3D DIC setup (VIC3D7 setup and software acquired from Correlated Solutions). Young's modulus and Poisson's ratio for the tested epoxy were then computed from the tensile test results and are presented in Table 1.

Four different reinforcements were used in this study: a single nickel plated high carbon steel fiber (HCS), a single polylactic acid (PLA) fiber, a single polytetrafluoroethylene (PTFE) fiber and a bundle of carbon fibers (CF). The HCS fibers were a $200 \mu \mathrm{m}$ diameter wires manufactured by D'addario and company Inc. The PLA-4032D fibers were manufactured using the solvent assited 3D-printing method [31] with a $200 \mu \mathrm{m}$ diameter nozzle. The fiber's final diameter was $180 \mu \mathrm{m}$ due to material evaporation after extrusion. The material was acquired as pellets from Nature Works LLC. The PTFE fibers were $711 \mu \mathrm{m}$ diameter monofiber filaments and were acquired from Zeus Inc. Note that we did not succeed at acquiring PTFE wires having

Table 1 Studied materials elastic properties

\begin{tabular}{llll}
\hline Material & $\begin{array}{l}\text { Young's Modulus } \\
(\mathrm{GPa})\end{array}$ & Poisson's ratio & $\begin{array}{l}\text { Surface energy } \\
(\text { dynes/cm })\end{array}$ \\
\hline Epoxy & $2.45 \pm 0.90$ & 0.46 & 45 \\
PTFE & $0.390-0.600$ & 0.46 & 19 \\
PLA & $2.70 \pm 0.69$ & 0.36 & 38 \\
HCS & 200 & 0.29 & Strong \\
CF & - & - & $70[30]$ \\
\hline
\end{tabular}

Epoxy and PLA's Young's Modulus and Poisson's ratio were measured experimentally according to the ASTM D638 standard. The \pm corresponds to a $95 \%$ confidence interval on the mean value. The PTFE and HCS's properties were provided as a range by their respective manufacturers 
Fig. 2 Schematic illustration of the LSCM paired with the microtensile device for in-situ full field strain measurements. The specimen is mounted into the micro-tensile test machine which is installed on the LSCM plate. The microscope's lens is then aligned with the fiber. The load is applied along the $y$ direction and is shown with two black arrows. The microtensile device applies displacement to the specimen while measuring the force thanks to a load cell. Images acquired by the LSCM are saved on a computer for later $\mu \mathrm{DIC}$ analysis

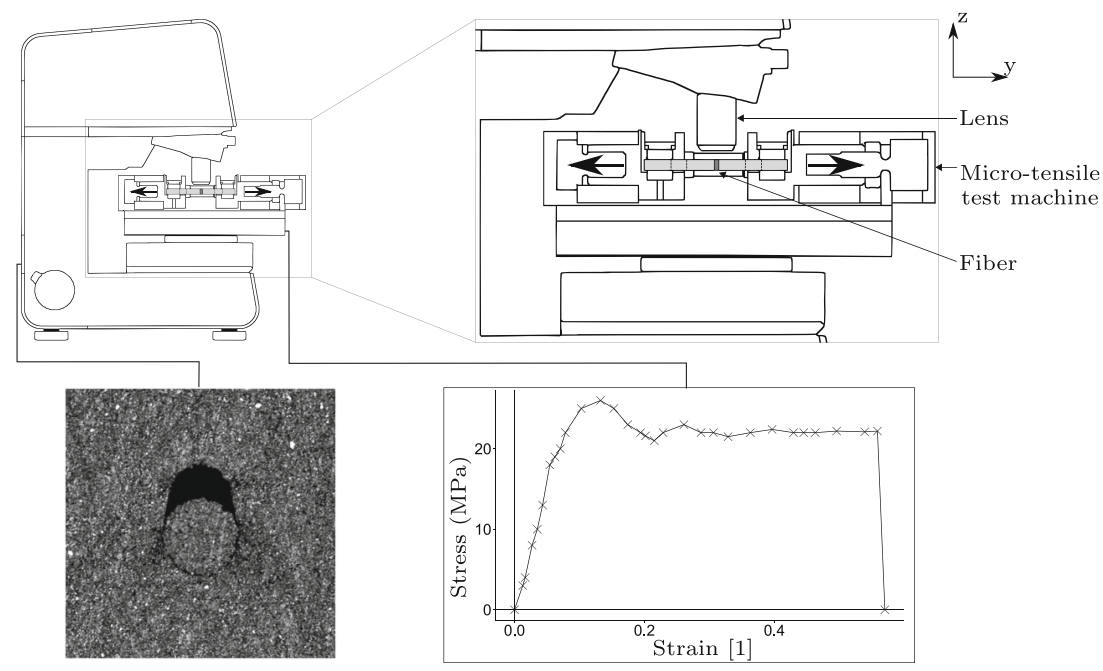

the same diameters as the other fibers. A bundle of fibers was cut from an Injectex GF420-E01-100 carbon fabric manufactured by Hexcel. Fiber diameters were measured using a LSCM for 30 fibers and the average diameter was found to be $7.5 \mu \mathrm{m} \pm 0.2$. The $\mathrm{CF}$ were impregnated with the resin and hardener mixture prior to being deposited into the mold to improve bonding and minimize defects within the bundle.

Table 1 lists the elastic properties measured or obtained from each manufacturer. PTFE is a non-reactive synthetic polymer with a very low surface energy of $19 \frac{\text { dynes }}{\mathrm{cm}}$. This surface energy is not sufficiently high to create bonds with epoxy [32]. A surface energy higher than $30 \frac{\text { dynes }}{\mathrm{cm}}$ is usually required to bond with a cured epoxy [33]. Thus, a PTFE fiber inside an epoxy matrix can only be held through compressive forces due to the difference in coefficients of thermal expansion (CTE) between the matrix and fibers, and epoxy's shrinkage resulting from its curing process [34]. PLA's surface energy is slightly higher than PTFE's, $38 \frac{\text { dynes }}{\mathrm{cm}}$, which is sufficient to create a weak bonding with epoxies [35]. HCS has a surface energy of several hundred dynes and strongly adheres to epoxy [32]. An average surface energy for unsized carbon fibers was reported to be about $70 \frac{\text { dynes }}{\mathrm{cm}}[30,36]$. No sizing was applied on the CFs.

\section{Methods}

\section{Experimental Setup}

The experimental setup used in this work is schematically shown in Fig. 2.

Digital images with a resolution of $4096 \times 4096 \mathrm{px}^{2}$ were acquired with a LEXT OLS4100 Laser Scanning Confocal Microscope, manufactured by Olympus $@$, while the specimen was loaded using a $5 \mathrm{kN}$ Kammrath and Weiss micro tensile device.

The specimen was deformed step-by-step with a regular macroscopic displacement increment $\Delta \mathrm{V}$ of approximately $50 \frac{\mu \mathrm{m}}{\mathrm{min}}$. At each step, the cross-head's displacement was held, and sufficient time was allowed $(\approx 1 \mathrm{~min})$ prior to image capture to let the specimen fully relax. The load was continuously recorded during the mechanical tests. The LSCM was equipped with a dual confocal system to limit out of plane measurements artifacts for composites with different reflecting characteristics.

\section{Speckle pattern}

Under the LSCM, local topographic variations induce local changes in reflectivity, depicted as grey levels using the
Table 2 Fiber diameter, size of the area observed and scale for each specimen studied

\begin{tabular}{lllll}
\hline Specimen & $\begin{array}{l}\text { Fiber diameter } \\
(\mu \mathrm{m})\end{array}$ & $\begin{array}{l}\text { Observation area } \\
(\mu \mathrm{m} \times \mu \mathrm{m})\end{array}$ & $\begin{array}{l}\text { Scale } \\
\left(\frac{\mathrm{px}}{\mu \mathrm{m}}\right)\end{array}$ & $\begin{array}{l}\text { Height pitch } \\
(\mu \mathrm{m})\end{array}$ \\
\hline Epoxy / PTFE & 711 & $2560 \times 2560$ & 1.6 & 5.00 \\
Epoxy / PLA & 180 & $1280 \times 1280$ & 3.2 & 2.00 \\
Epoxy / HCS & 200 & $1280 \times 1280$ & 3.2 & 2.00 \\
Epoxy / CF & $7.5 \pm 0.2$ & $256 \times 256$ & 16.0 & 0.06 \\
\hline
\end{tabular}

The height pitch, height step between two out of plane measurements, is also provided 
Table 3 Subset and step parameters selected for each test analysis

\begin{tabular}{lll}
\hline Fiber & Subset & Step \\
\hline PTFE & 91 & 3 \\
PLA & 99 & 3 \\
HCS & 99 & 3 \\
CF bundle & 93 & 3 \\
\hline
\end{tabular}

laser intensity micrographs with sufficient contrast for DIC analysis [37, 38].

After being mirror polished with silicon-carbide grit paper up to P4000 grit, each specimen was gently polished manually in random directions for a dozen seconds around the fiber with P2400 and P1000 grit papers to produce random and very fine scratches intercepting together to form the speckle pattern.

Polishing was carried out incrementally and observed under the LSCM at each step to ensure that: (i) no large scratches were observed on the Region of Interest (ROI), (ii) a sufficiently large array of grey levels was visible in the ROI to distinguish every subset.

Table 2 lists the observed area, scale, and height pitch for each specimen. The height pitch is the step used between two depth scans of the LSCM and represents the precision of the out of plane measurements' precision.

\section{Selection of DIC parameters}

A subset is a square zone centered around a given pixel the software is currently tracking and its size is expressed as a number of pixels.

In subset based DIC, a subset from the initial image (reference image) is compared with subsets from the current image (deformed image) until a subset better matching the initial image is found. Subsets are compared using a correlation function. The larger eigenvalue of the covariance matrix of the correlation equation is used to obtain the confidence margins, $\mathcal{C}$, for the displacements estimated by DIC for each pixel [37]. This value is representative of the error between between a reference and deformed
Fig. 3 PTFE/epoxy single-fiber composite loaded in tension in the $y$ direction. Column (a) includes images taken before an interfacial crack appeared between the fiber and matrix. Column (b) includes images taken after an interfacial crack appeared. Column (c) includes images taken before DIC tracking was lost. The global stress and strain values for each column are provided at the very top of the figure. The first row shows the raw images. The second and third rows present the strain contour plots in the $y$ and $x$ directions, and the last row presents the shear strain $x y$ a
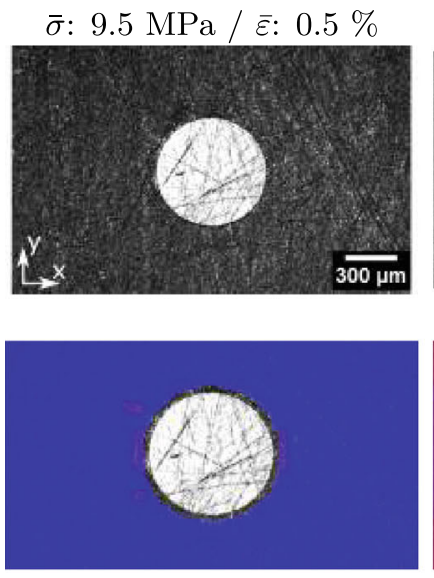

0

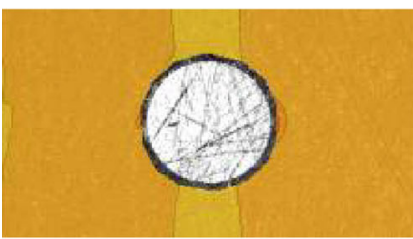

$-7$

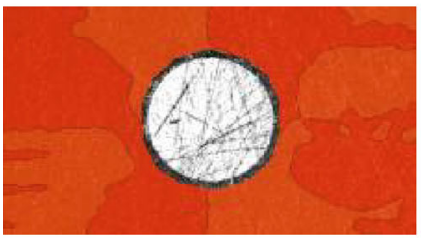

$-3.5$ b $\bar{\sigma}: 20.5 \mathrm{MPa} / \bar{\varepsilon}: 2.3 \%$
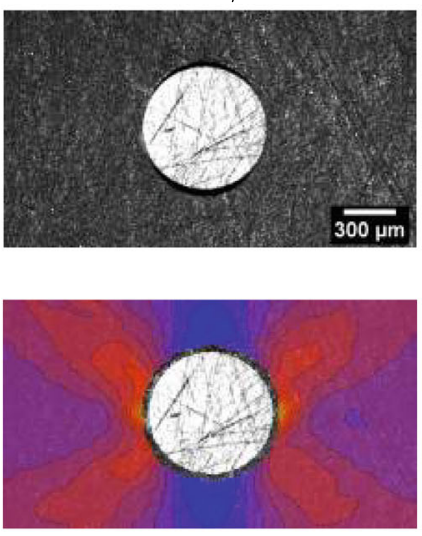

$\varepsilon_{y}(\%)$

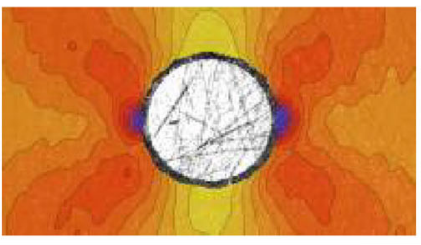

$\varepsilon_{x}(\%)$

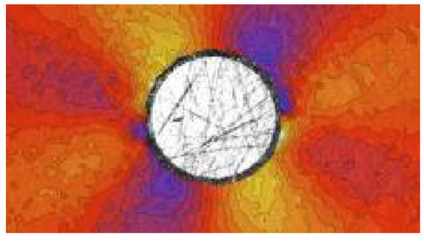

$\varepsilon_{x y}(\%)$ c $\bar{\sigma}: 24.9 \mathrm{MPa} / \bar{\varepsilon}: 3.4 \%$
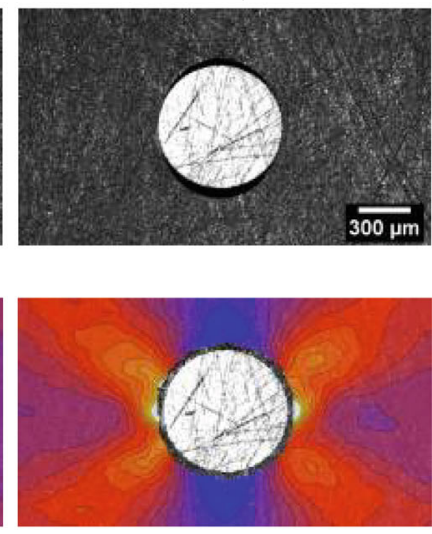

42 
subset [38]. This information can then be used to measure the subset's displacement at a subpixel resolution, and thus the whole ROI's displacement field.

The step size is defined as the spacing between the points analyzed during correlation. Displacement values for pixels that are not tracked by the software (between two consecutive steps) are interpolated between tracked pixels, assuming a continuous displacement gradient [38]. A step of 2 means that one pixel out of two is tracked, with the displacement of the others being interpolated [38]. A step of size 2 was chosen to minimize the area lost around the crack and to minimize smoothing effects around discontinuities created by to interpolation [37].

Lagrangian strains were computed using a filter size of 5 pixels to avoid over-smoothing the experimental data while computing strains. The strain computations use a filter size of 5 pixels. With the step set to 2 , this means that the total smoothing area due to strain computation is of 10 pixels.
At some point, a subset distortion is so large that the confidence is too large to ensure pixel tracking. Features like the apparition of cracks typically lead to a loss of tracking [38].

A subset was considered untracked when the confidence margin exceeded an arbitrary threshold value of 0.1 , as defined in VIC3D. Pixels for which tracking was lost were represented as white in the contour plots.

Large steps lead to faster computation, when compared to smaller steps, but decrease the measurements accuracy. However, small steps can induce large noise in strain computations. The optimal choice of subset and step is therefore a trade-off between the above mentioned beneficial and detrimental effects [37, 38].

Table 3 lists the subsets and steps that were used for displacement measurements using the subset suggestion tool available in VIC2D commercial software. The program computes the ideal subset size for an optimal match confidence of 0.01 pixel [38].
Fig. 4 PLA/epoxy single-fiber composite loaded in tension in the $y$ direction. Column (a) includes images taken before an interfacial crack appeared between the fiber and matrix. Column (b) includes images taken after an interfacial crack appeared. Column (c) includes images taken before DIC tracking was lost. The global stress and strain values for each column are provided at the very top of the figure. The first row shows the raw images. The second and third rows present the strain contour plots in the $y$ and $x$ directions, and the last row presents the shear strain $x y$ a

$$
\bar{\sigma}: 6.3 \mathrm{MPa} / \bar{\varepsilon}: 0.5 \%
$$

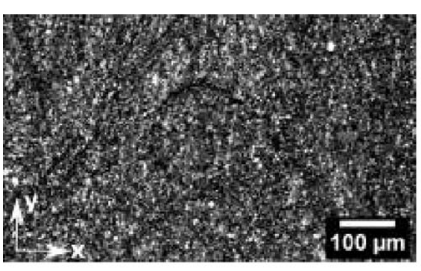

$\bar{\sigma}: 13.2 \mathrm{MPa} / \bar{\varepsilon}: 4.9 \%$

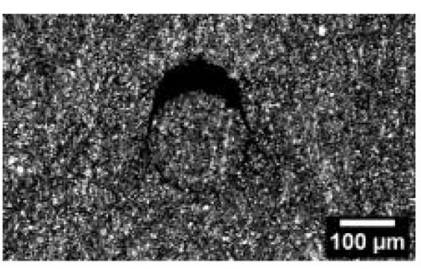
$\bar{\sigma}: 13.5 \mathrm{MPa} / \bar{\varepsilon}: 7.0 \%$
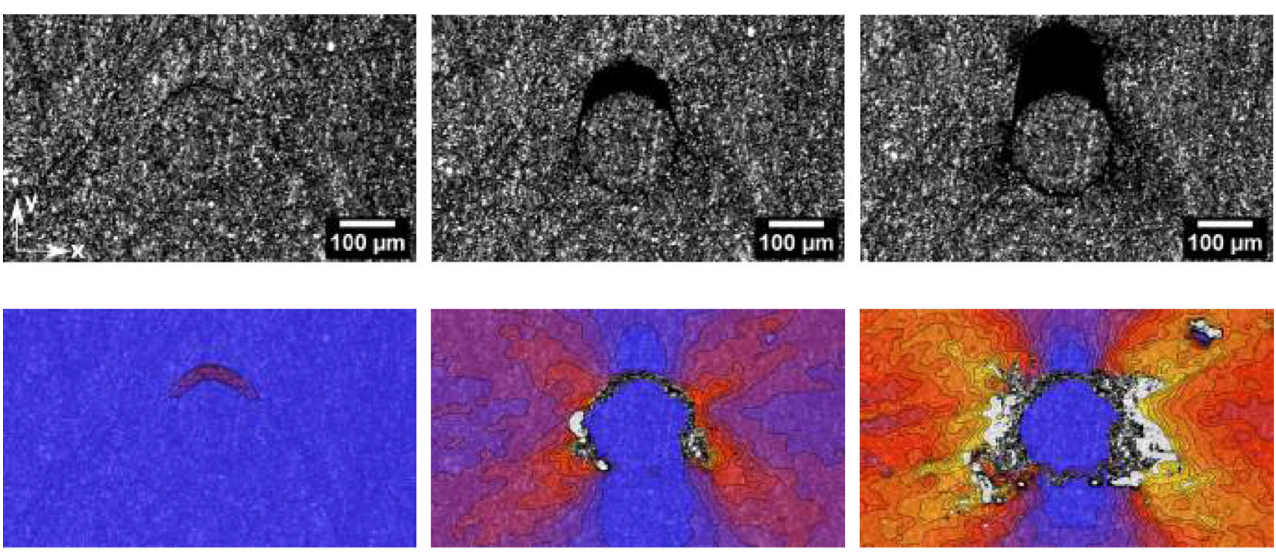

0

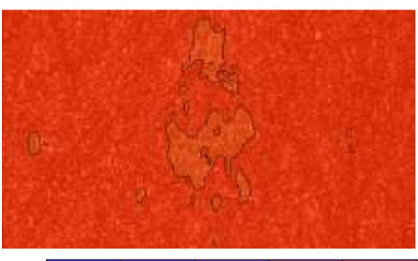

$-15$

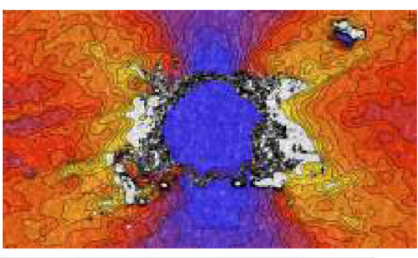

$\varepsilon_{y}(\%)$ 42

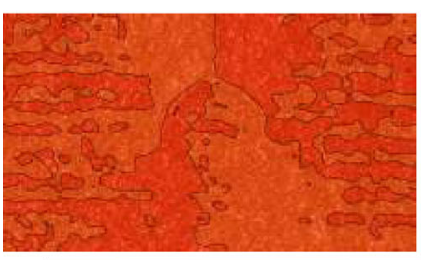

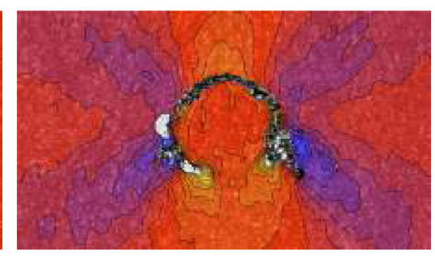

$\varepsilon_{x}(\%)$

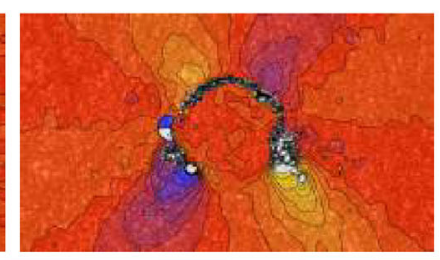

$\varepsilon_{x y}(\%)$

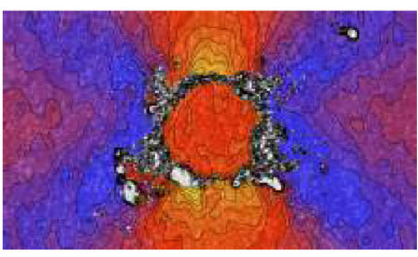
15

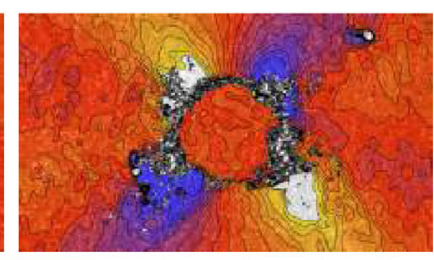

$-20$ 
The DIC analysis for full field measurements was an in plane analysis. Out of plane measurements were simultaneously recorded when capturing the laser intensity micrographs. A stabilization algorithm was applied on each image to remove the microscope's rigid body motion during the experiment prior to DIC analysis.

\section{Results}

\section{Single Fiber}

$\varepsilon_{x}, \varepsilon_{y}$ and $\varepsilon_{x y}$ contour plot time-lapses for the PTFE / epoxy, PLA / epoxy and HCS /epoxy specimens are respectively shown in Figs. 3, 4 and 5.

The reference coordinate axes used $(x, y$ and $z)$ are presented in Figs. 1 and 2. Strain measurements were provided in PLA fibers while it was not possible to accurately measure strains in the HCS and PTFE fibers due to their highly reflective surfaces that saturated the fibers with white pixels.

\section{Strain evolution}

Strain component $\varepsilon_{y}$ localized at the $90^{\circ}$ and $-90^{\circ}$ areas for the PTFE / epoxy specimen (see Fig. 3(a)). For the PLA / epoxy specimen, $\varepsilon_{y}$ localized around the $0^{\circ}$ and $180^{\circ}$ interfacial areas (see Fig. 4(a)).

The maximum $\varepsilon_{y}$ recorded at this location was about twice the applied strain for the PTFE / epoxy specimen and more than seventeen times higher for the PLA / epoxy specimen. Figure 3(a) also shows that a crack appeared at the top and bottom of the PTFE / epoxy interface for $\bar{\varepsilon}=0.5 \%$. An interfacial crack is also clearly visible at the top of the PLA fiber, where $\varepsilon_{y}$ localized (Fig. 4(a)). The HCS / epoxy specimen also exhibits a large interfacial crack extending from $135^{\circ}$ to $-90^{\circ}$ (Fig. 5(a)). The $\varepsilon_{x}$ and $\varepsilon_{x y}$ strain components (Figs. 3(a), 4(a), and 5(a)) are relatively smaller, when compared to $\varepsilon_{y}$ at the beginning of the tests.

As the load increases, the interfacial crack located at the top and bottom of the PTFE fiber keeps growing. Figure 3(b) shows how $\varepsilon_{y}$ builds up into a cross shape centered on the PTFE fiber with the highest $\varepsilon_{y}$ strain located b

$\bar{\sigma}: 14 \mathrm{MPa} / \bar{\varepsilon}: 5.6 \%$
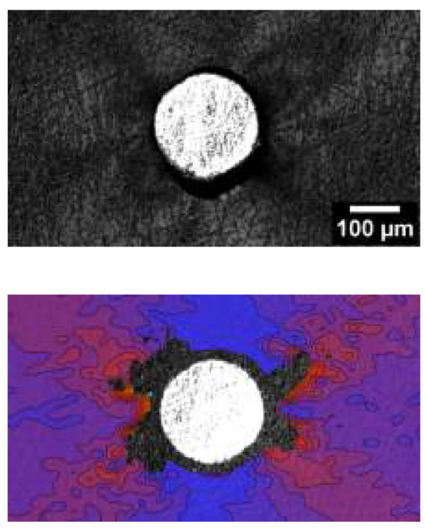

$\varepsilon_{y}(\%)$

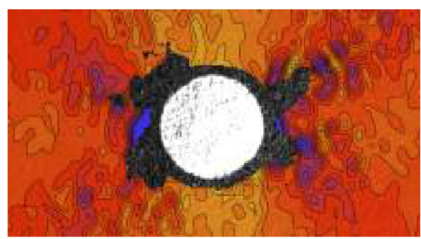

$\varepsilon_{x}(\%)$

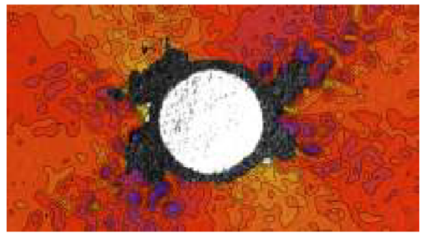

$\varepsilon_{x y}(\%)$ c

$$
\bar{\sigma}: 14.2 \mathrm{MPa} / \bar{\varepsilon}: 9 \%
$$
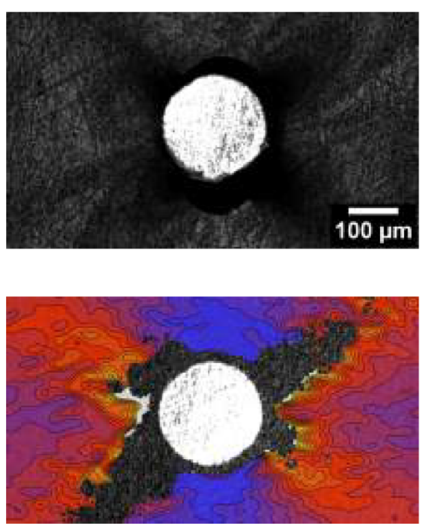

45

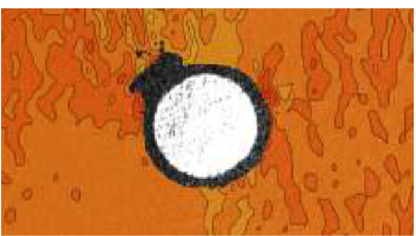

$-15$

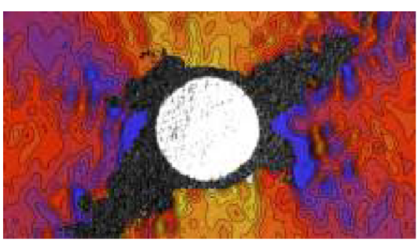

7.5

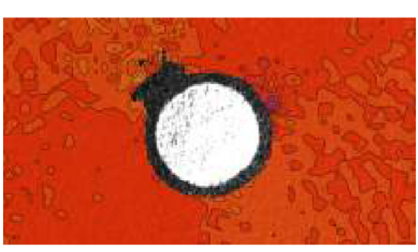

$-12$

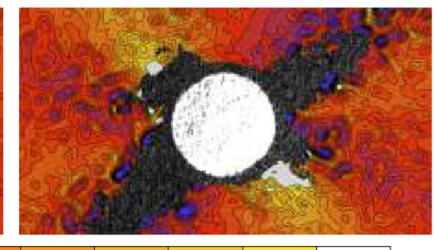


along the diagonals $\left(45^{\circ}, 135^{\circ},-135^{\circ}\right.$, and $\left.-45^{\circ}\right)$. The $\varepsilon_{y}$ maximum eventually localized at the interface along the $+90^{\circ}$ and $-90^{\circ}$ interfacial areas. $\varepsilon_{x}$ also forms a cross shape around the fiber, with its peak located at the top and bottom of the fiber. The cross shape encompasses a gradient of compressive strains, with the maximum $(-7 \%)$ precisely localizing at the $-90^{\circ}$ and $+90^{\circ}$ areas around the interface. $\varepsilon_{x y}$ is symmetrically distributed around the fiber with its maximum $(3.5 \%)$ localized around the $-135^{\circ}$ and $+45^{\circ}$ areas, while the minimum $(-3.5 \%)$ is located around the $135^{\circ}$ and $-45^{\circ}$ areas. $\varepsilon_{x y}$ is close to 0 next to the $0^{\circ}$ and $180^{\circ}$ areas around the fibers while the extrema are located around the $45^{\circ}, 135^{\circ},-135^{\circ}$ and $-45^{\circ}$ interfacial areas. Figure 3(c) shows that the $\varepsilon_{y}, \varepsilon_{x}$ and $\varepsilon_{x y}$ fields exhibit a similar shape as the load increases. Both cracks $\left(0^{\circ}\right.$ and $\left.180^{\circ}\right)$ keep growing with that at $180^{\circ}$ becoming slightly longer.

The PLA / epoxy specimen's interfacial crack opened at $0^{\circ}$. The top crack is about $60 \mu \mathrm{m}$ long in Fig. 4(b). An interfacial crack is also growing all around the fiber. The top crack reached a length of up to $130 \mu \mathrm{m}$ for a global strain applied of 7\%, as shown in Fig. 4(c). Short lateral cracks are visible in the matrix next to the interface at $-100^{\circ}$ and $100^{\circ}$. Other cracks, which are opening in Mode I, are also visible along the top interfacial crack's right hand side. DIC tracking is gradually lost along the crack path. The behavior of $\varepsilon_{y}$ for the PLA fiber is similar to that described for the PTFE / epoxy specimen: the strain levels are similar, except for the maximum, which is spread over larger areas between $\left[45^{\circ} ; 135^{\circ}\right]$ and $\left[-135^{\circ} ;-45^{\circ}\right]$ around the interface (Fig. 4(c)).

The $\varepsilon_{x}$ strain field in the PLA fiber is similar to that observed in the PTFE / epoxy specimen. Although the minimum of $\varepsilon_{x}(-9 \%)$ is also located around the interface at the $90^{\circ}$ and $-90^{\circ}$, the area over which it spreads is larger than that observed in the PTFE / epoxy specimen. The $\varepsilon_{x y}$ field for the PLA / epoxy specimen is also similar to that observed for the PTFE / epoxy sample. Figure 4(c) shows that, although the crack is visible all around the fiber, only the top interfacial crack keeps growing and is as wide as the fiber itself, for $\bar{\varepsilon}=7.0 \%$.

By opposition, for the HCS / epoxy specimen, untracked pixels appeared early on during the test. The area over which untracked pixels appeared throughout the test is the cross shaped area over which $\varepsilon_{x}$ was the most compressive and $\varepsilon_{y}$ was high for the other specimens, as shown in Figs. 5(b) and 5(c). The $\varepsilon_{y}, \varepsilon_{x}$ and $\varepsilon_{x y}$ strain fields have distributions similar to those measured in the PLA / epoxy and PTFE / epoxy specimens.

\section{Tangential strain}

We define $\varepsilon_{y}^{T}$ as the averaged $\varepsilon_{y}$ strain measured over two discs on the right $\left(-90^{\circ}\right)$ and left $\left(90^{\circ}\right)$ hand side of each fiber. Figure 6 shows $\varepsilon_{y}^{T}$ measurements around the fiber, for each specimen. While the top and bottom crack open under Mode I, the cracks on the left and right sides of fibers open under Mode II, as shown by the $\varepsilon_{y}$ fields presented in Figs/ 3, 4, and 5. $\varepsilon_{y}^{T}$ represents tangential opening which induces Mode II opening [19]. $\varepsilon_{y}^{T}$ growth rate is similar for every sample up to around $3.5 \%$ global strain. After this threshold, $\varepsilon_{y}^{T}$ grows rapidly in the PLA / epoxy and PTFE / epoxy specimens, while its growth is significantly slower in the HCS / epoxy specimen. This behavior suggests that tangential debonding occurred much faster in the PLA / epoxy and PTFE / epoxy specimens than in the HCS / epoxy specimen, which is consistent with the interfacial strength reported in Table 1.

\section{Virtual extensometers}

Figure 7 shows the strain that would have been measured by an extensometer located near the fiber / matrix interface as

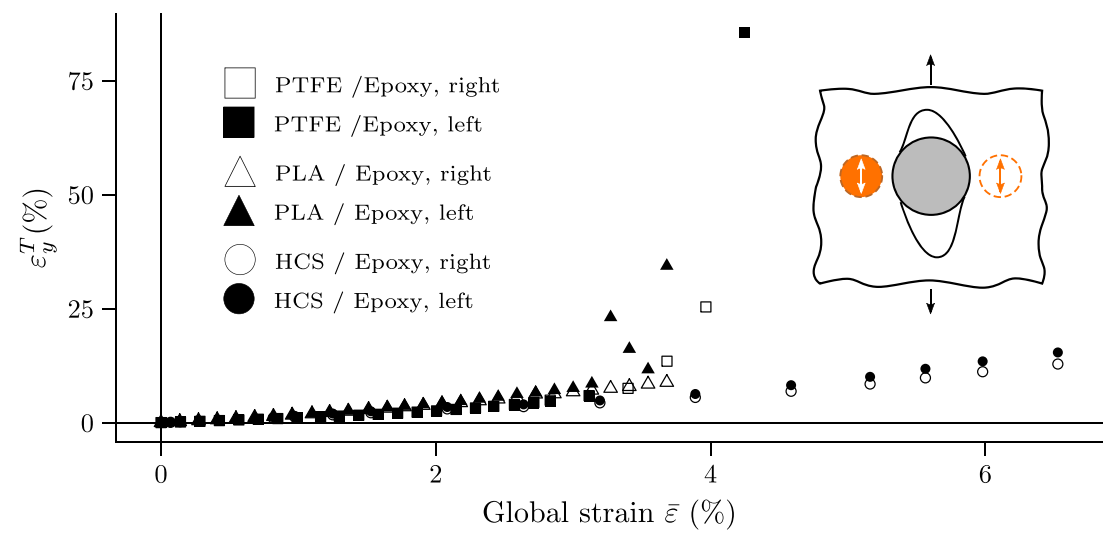

Fig. 6 The average strain $\varepsilon_{y}^{T}$ is plotted for two positions around the fiber, for each specimen. The average strains of pixels within two discs about half a subset away from each fiber were computed and plotted. Each disc is about $\frac{2}{3}$ of the size of the fiber it is next to. $\varepsilon_{y}^{T}$ growth rate is about the same for all three specimens before $3 \%$ global strain. It then strongly increases for the PLA / epoxy and PTFE / epoxy specimens, while it increases steadily for the HCS 


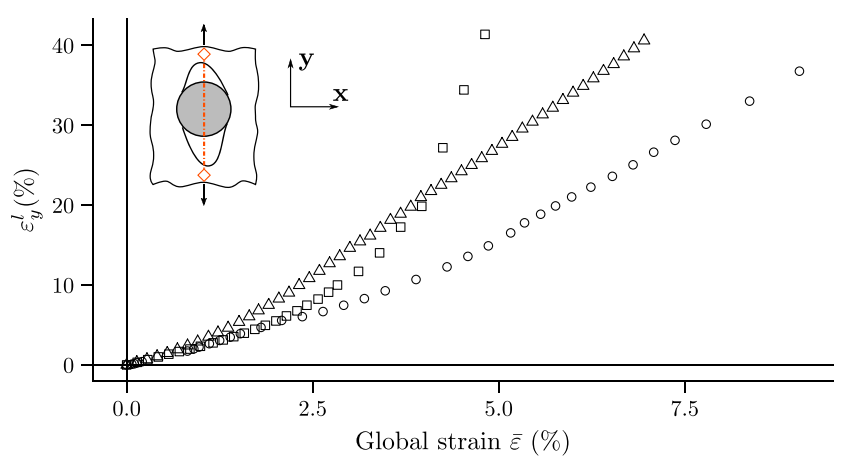

a

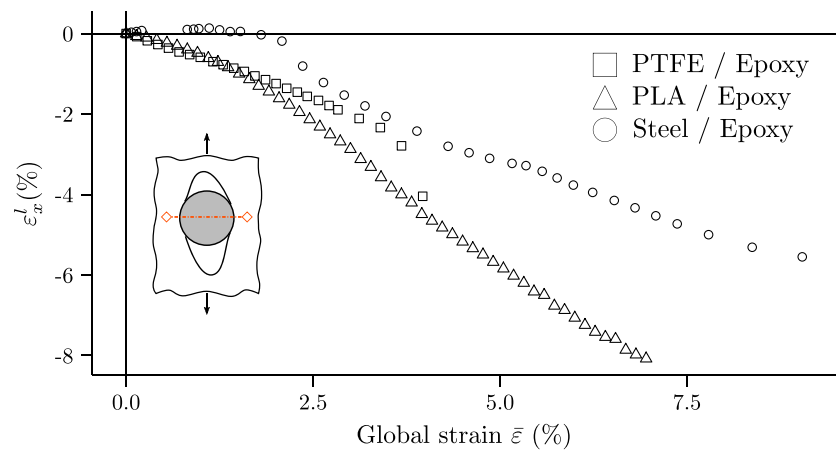

a

Fig. $7 \varepsilon_{x}^{l}$ and $\varepsilon_{y}^{l}$ are shown as a function of the global strain applied on the specimen $\bar{\varepsilon} . \varepsilon_{x}^{l}$ and $\varepsilon_{y}^{l}$ are obtained by applying a virtual extensometer around the fibre/matrix interface, as shown on the schematics in Figs. (a) and (b). $\varepsilon_{x}^{l}$ measures the strain applied by the matrix on the fiber along the $x$ direction. $\varepsilon_{y}^{l}$ measures the strain applied by the matrix on the fiber and the opening of the interfacial fiber, along the $y$ direction

a function of the global strain $\bar{\varepsilon}$. Two pixels were selected about a subset away from the fiber / matrix interface, in the $x$ and $y$ directions, for every specimen. The local strain was computed from the pixels displacement as: $\varepsilon^{l}=\frac{\Delta L}{L_{0}}$, where $L_{0}$ is the initial distance between the two selected pixels and $\Delta L$ the distance variation between both pixels. $\varepsilon_{y}^{l}$ was measured along the $y$ direction, and $\varepsilon_{x}^{l}$ along the $x$ direction. $\varepsilon_{y}^{l}$ provides insights on the interfacial crack opening along the $y$ direction.

Figure 7(a) shows the strain measured by the virtual extensometer aligned along the $y$ direction near the fiber / matrix interface. Although $\varepsilon_{y}^{l}$ grows with about the same rate for every specimen, its opening rate in PTFE / epoxy and PLA / epoxy specimens accelerates after around 1\% while it remains constant for the HCS specimen up to about $3 \%$. Note that $\varepsilon_{y}^{l}$ 's opening rate is slitghly faster in the PLA / epoxy specimen than that measured in the PTFE / epoxy specimen. This behavior is consistent with the PTFE / epoxy bonding strength.

After $\bar{\varepsilon} \approx 2.5 \%, \varepsilon_{y}^{l}$ in the PTFE / epoxy specimen significantly increases up to $40 \%$, opening much more and faster than what is observed in the PLA / epoxy specimen.
The $\varepsilon_{y}^{l}$ strain rate in the HCS / epoxy and the PLA / epoxy specimens gradually increases quasi linearly, with the opening rate for the PLA / epoxy specimen faster that of the HCS / epoxy specimen. The PLA / epoxy specimen has as weaker interfacial bonding that the HCS / epoxy specimen, which is consistent with their respective opening rates. The results quantitatively show that $\varepsilon_{y}^{l}$ is affected by the fiber / matrix couple's bonding strength.

Figure 7(b) presents the strain measured by the virtual extensometer aligned along the $x$ direction near the fiber / matrix interface. The PTFE / epoxy and PLA / epoxy specimens $\varepsilon_{x}^{l}$ grow at the same rate up to an applied global strain of about $2 \%$. $\varepsilon_{x}^{l}$ measured for the PLA / epoxy specimen becomes more compressive than that observed in the PTFE / epoxy specimen. Moreover, $\varepsilon_{x}^{l}$ 's growth rate for the PTFE / epoxy specimen accelerates after a global strain of 3\%. The behavior of the HCS / epoxy specimen is significantly different. Under an applied strain rate of $2 \%, \varepsilon_{x}^{l}$ is slightly positive or about $0 \%$, showing that the extensometer marginally undergoes tension at first along the $x$ axis. It then becomes compressive, and decreases quasilinearly with a slower rate than the two other specimens.

This behavior is attributed to the stronger bonding of the HCS fiber with the epoxy matrix.

\section{Out of plane measurements}

Out of plane measurements are independent of the DIC measurements as they were obtained solely using the data provided by the microscope.

Figure 8 presents $z(x)$ and $z(y)$ out of plane measurements along profile lines oriented in the $x$ and $y$ directions, at the last time step (corresponding to Figs. 3(c) and 4(c)). Figure 8 shows that each fiber is protruding above the matrix that underwent compression due to Poisson's effect, for all specimens.

Figure 8(a) shows $z(y)$ and $z(x)$ for the PTFE / epoxy specimen. $z(y)$ exhibits two gaps resulting from the $0^{\circ}$ and $180^{\circ}$ in plane interfacial cracks shown in Fig. 8(b). Two clear discontinuities can be seen in the $z(y)$ height profile (highlighted with blue dashed lines in Fig. 8(b)), showing that out of plane interfacial debonding clearly happened due to the tunneling effect. The difference in height between the fiber's free surface and the matrix - the out of plane interfacial crack - along the $x$ axis is about $390 \mu \mathrm{m}$ and is shown as a red dashed line in Fig. 8(b).

Figure $8(\mathrm{c})$ shows that a large in plane top crack $\left(0^{\circ}\right)$ is clearly visible and is about $200 \mu \mathrm{m}$ long in the PLA / epoxy specimen. Figure $8(\mathrm{~d})$ presents $z(y)$ and $z(x)$ for the PLA / epoxy specimen. Note that the portion of $z(y)$ 's profile where there is no reported value (shown as a blue dashed line) corresponds to the crack's location. Figure 8(c) also shows the $180^{\circ}$ interfacial crack which appears as a gradual 
Fig. 8 Height measurements along longitudinal and transverse profile lines for each single fiber specimen. The left hand side shows the fiber along with the $x$ and $y$ profile lines over which the $z$ coordinate is extracted. The right hand side shows the actual plots for the $z$ coordinates. Figures (a) and (b) present these results for the PTFE / epoxy specimen at the same global strain as Fig. 3(c); Figs. (c) and (d) for the PLA / epoxy specimen and Fig. (e) at the same global strain as Fig. 4(c) and (f) the HCS / epoxy at the same global strain as Fig. 5(c). Blue dashed lines were added to the plots to highlight in plane cracks and red dashed lines for the out of plane cracks $\mathrm{mm}$

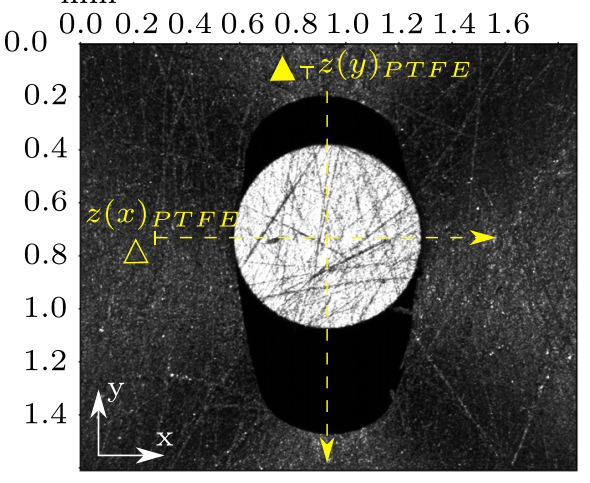

$\mathbf{a}$

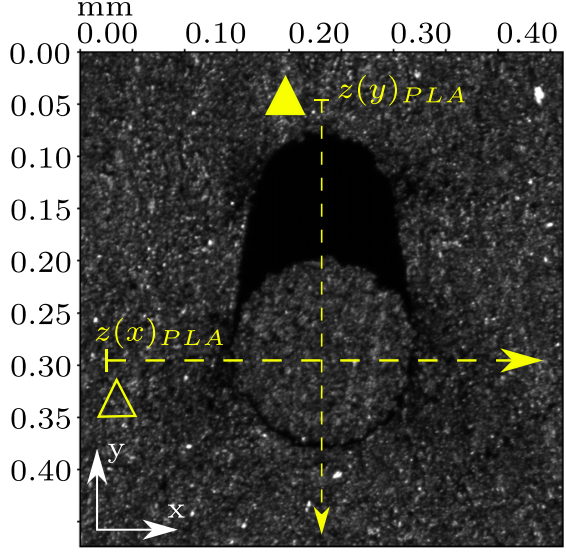

c

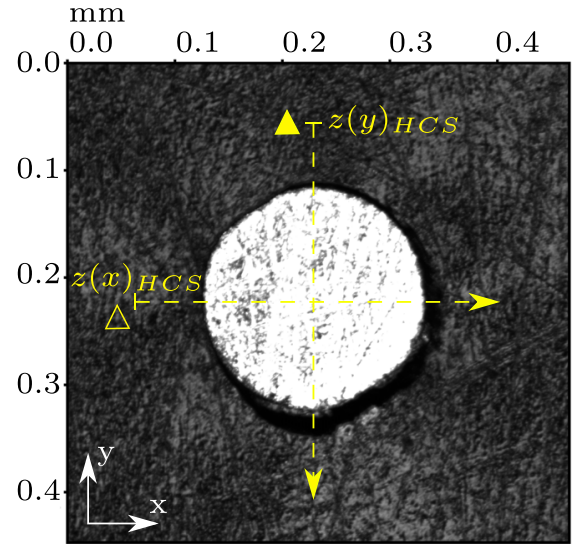

e

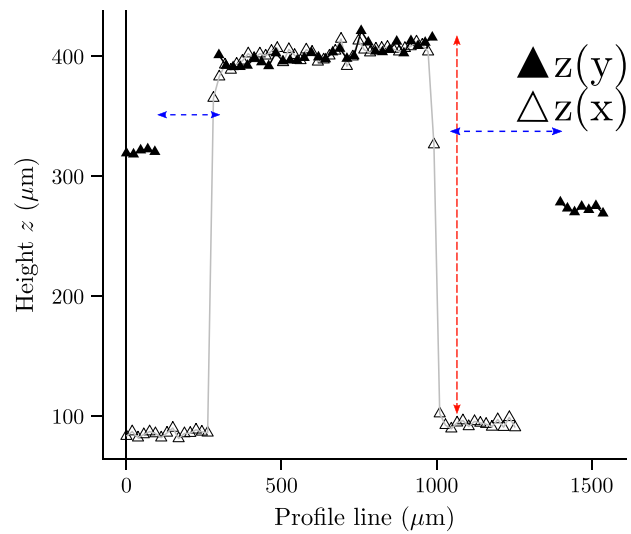

b

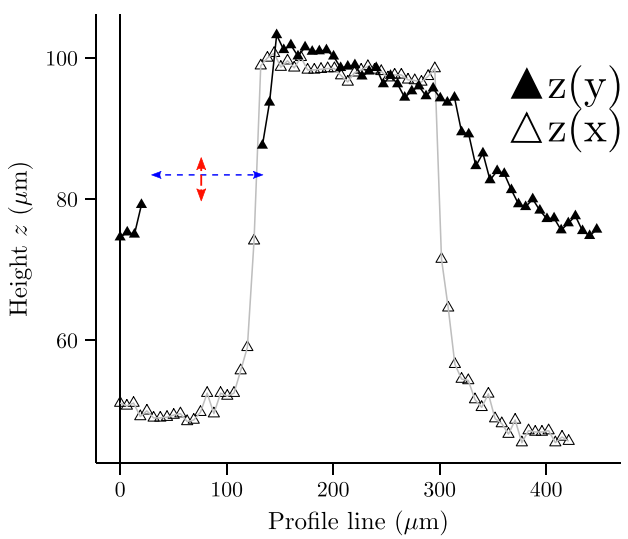

d

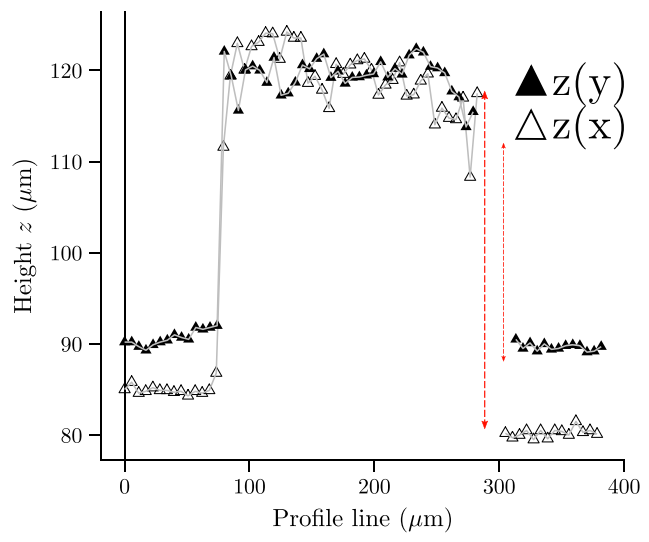

f height decrease, from $300 \mu \mathrm{m}$ onward. The fiber's top free surface appears to be tilted toward the $180^{\circ}$ crack. These two observations suggest that the fiber is still bonded to the matrix, only for angles ranging from $\approx 135^{\circ}$ to $-135^{\circ}$.

Figure 8(e) presents similar results for the HCS / epoxy specimen. An in plane interfacial crack is visible at $180^{\circ}$ and $-90^{\circ}$, as presented on the micrograph. $z(y)$ and $z(x)$ profiles show that the out of plane interfacial crack is more than $30 \mu \mathrm{m}$ wide (shown with red dashed lines), which is about three times larger than the in plane interfacial crack. The transition from the fiber to matrix is similar to the profiles presented for PTFE / epoxy (Fig. 8(f)), except that the in plane crack is less than $10 \mu \mathrm{m}$ long.

\section{Carbon Fiber bundle}

Figure 9 shows the CF / epoxy specimen's studied area. Two areas were selected as ROIs. The larger area (ROI 


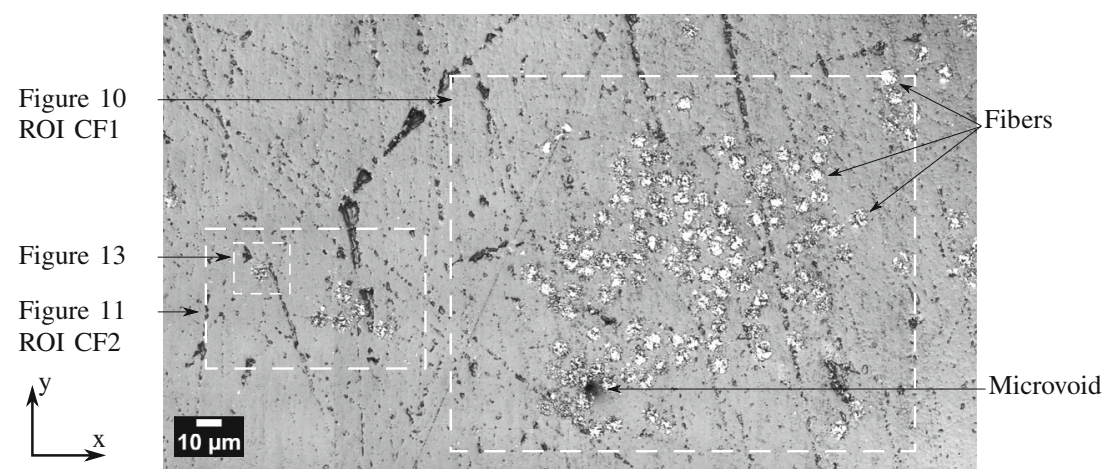

Fig. 9 Micrograph of the area of interest studied for the CF/epoxy. The area on the left contains a small bundle containing five carbon fibers and a single fiber on the top left. The area on the right contains a bundle of carbon fibers with about a hundred fibers. Within the area on the left, a single carbon fiber was arbitrarily selected to perform in plane and out of place crack size measurements, which are presented in Fig. 13

Fig. 10 Timelapse of CF bundle / epoxy composite loaded in tension in the $y$ for ROI CF1 in Fig. 9. The bundle contains about 100 CFs. Column (a) includes images taken before any damage is visible on the image. Column (b) includes images taken after interfacial cracks started appearing. Column (c) includes images taken before DIC tracking was lost. The global stress and strain values for each column are provided at the very top of the figure. The first row shows the raw images. The second and third rows present the strain contour plots in the $y$ and $x$ directions, and the last row presents the shear strain $x y$ a
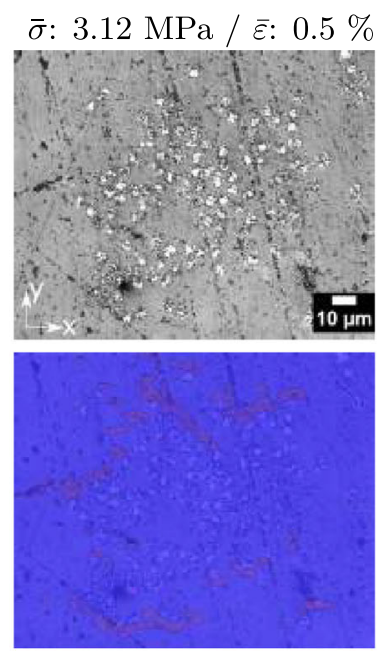

0

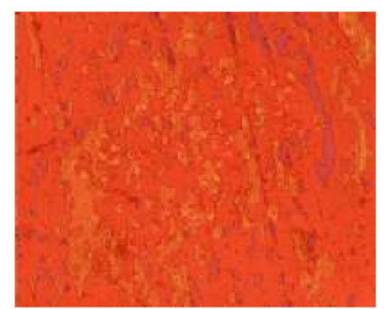

$\varepsilon_{y}(\%)$

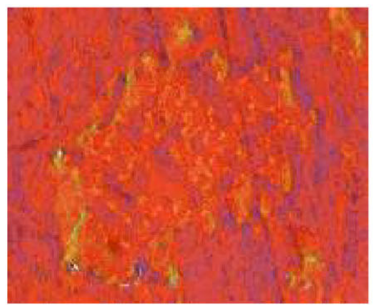

$-5$

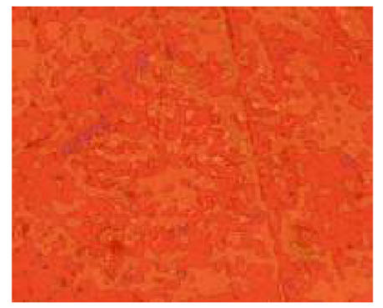

$\varepsilon_{x}(\%)$

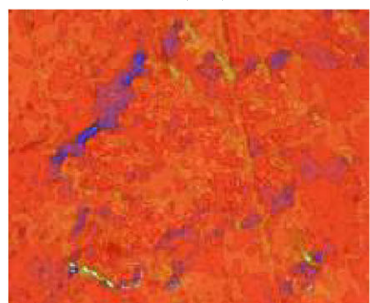

$-4$
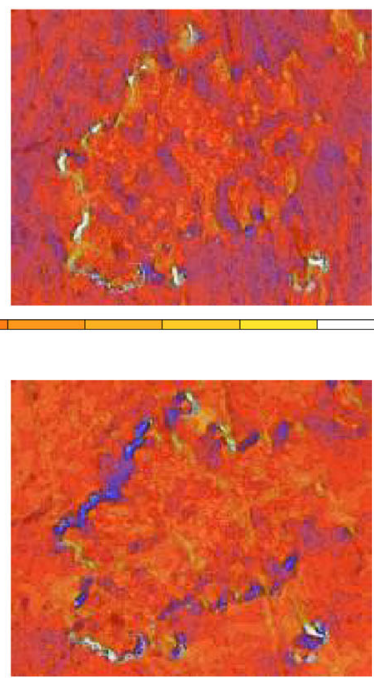

c $\bar{\sigma}: 7.7 \mathrm{MPa} / \bar{\varepsilon}: 2.5 \%$
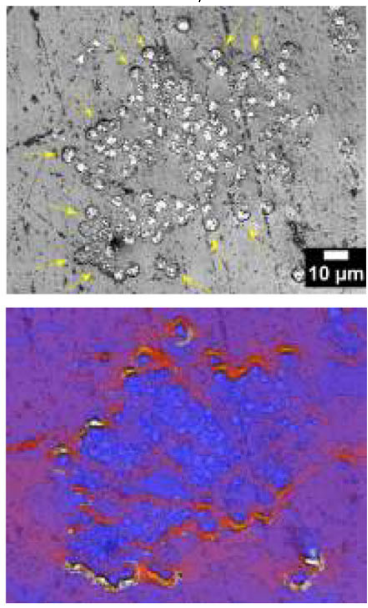

18

5

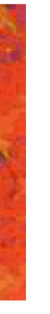

4 
CF1) contains about one hundred CFs loosely gathered in a bundle. A surface manufacturing defect is also visible in ROI CF1 (dark area). The second area (ROI CF2), on the left, shows a small bundle containing five CFs and a single fiber isolated on the top left. Images were acquired when the confocal microscope's plate was moved from one area to the other after the mechanical loading pauses.

\section{Strain evolution and damage}

Figures 10 and 11 show $\varepsilon_{x}, \varepsilon_{y}$ and $\varepsilon_{x y}$ strain field contour plots for ROI CF1 and ROI CF2, respectively, for arbitrarily chosen load levels.

The first row of Fig. 10 shows how the strain localizes and that damage develops around a relatively large bundle of CFs. Damage appears as interfacial cracks growing at the bundle's top and bottom. It is worth noting that the macroscopic crack at the bundle's top $\left(0^{\circ}\right)$ and bottom $\left(180^{\circ}\right)$ developed due to the coalescence of individual interfacial debonding cracks. Damaged interfaces are locared in the bundle's periphery along the $90^{\circ}$ and $-180^{\circ}$ areas, which is similar to what was observed in single fiber composites when the bundle is assimilated as a single fiber. These cracks are highlighted in Figs. 10(b) and 10(c) as yellow arrows. Similarly to the single fiber, $\varepsilon_{y}$ 's maximum is localized along the $0^{\circ}$ and $-180^{\circ}$ edges of the bundle. $\varepsilon_{x}$ 's maximum and minimum is localized along the $90^{\circ}$ and $-90^{\circ}$ areas. Figures $10(\mathrm{~b})$ and 10 (c) also show that $\varepsilon_{x}, \varepsilon_{y}$ and $\varepsilon_{x y}$ fields remain close to $0 \%$ within the bundle's core and exhibit concentrations along its edge. $\varepsilon_{y}$ 's maximum value is about 6 times higher than the global applied strain in Fig. 10(c).

Figure 11 shows the $\varepsilon_{y}, \varepsilon_{x}$ and $\varepsilon_{x y}$ fields for ROI $\mathrm{CF} 2$. Similarly to the larger $\mathrm{CF}$ bundle, damage initiates with debonding around the bundle's $0^{\circ}$ and $-180^{\circ}$ edges (Fig. 11(b)). Pixel tracking is gradually lost around the small bundle and the single fiber due to the apparition of interfacial cracks. The single fiber isolated on the top left corner of ROI CF2 is also, at this point, experiencing partial debonding. The $\varepsilon_{x}$ and $\varepsilon_{y}$ strain fields around the single fiber are similar to those observed for the single fiber
Fig. 11 CF bundle / epoxy single-fiber composite loaded in tension in the $y$ direction for ROI CF2 in Fig. 9. The bundle contains five $\mathrm{CFs}$ and an isolated fiber. Column (a) includes images taken before any damage is visible. Column (b) includes images taken after interfacial cracks appeared. Column (c) includes images taken before DIC tracking was lost. The global stress and strain values for each column are provided at the very top of the figure. The first row shows the raw images. The second and third rows present the strain contour plots in the $x$ and $y$ directions, and the last row presents the shear strain $x y$ $\bar{\sigma}: 3.12 \mathrm{MPa} / \bar{\varepsilon}: 0.5 \%$
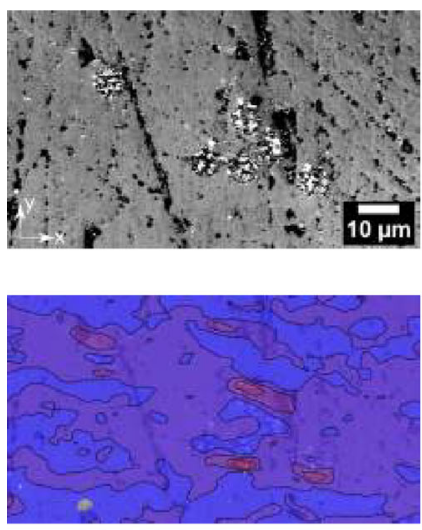

0

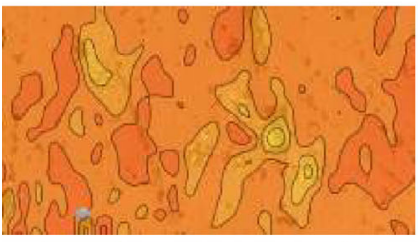

$-5$

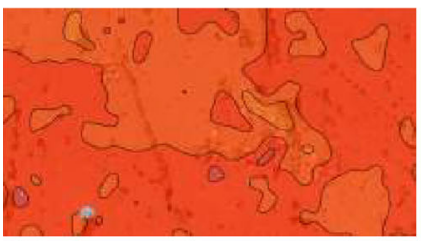

$-4$ b

$\bar{\sigma}: 4.5 \mathrm{MPa} / \bar{\varepsilon}: 0.9 \%$
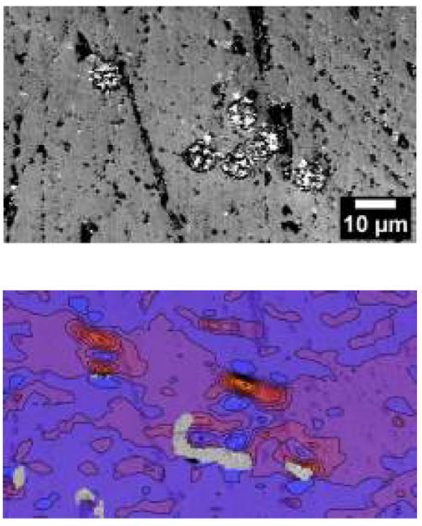

$\varepsilon_{y}(\%)$

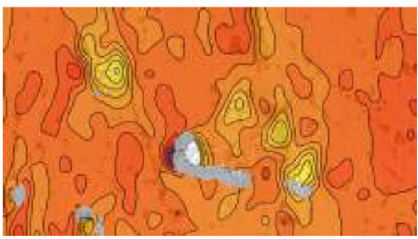

$\varepsilon_{x}(\%)$

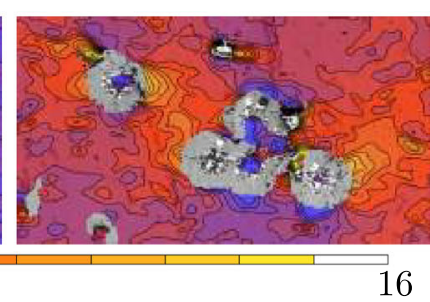

s

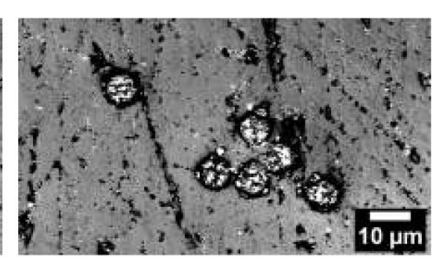

$\bar{\sigma}: 7.7 \mathrm{MPa} / \bar{\varepsilon}: 2.5 \%$

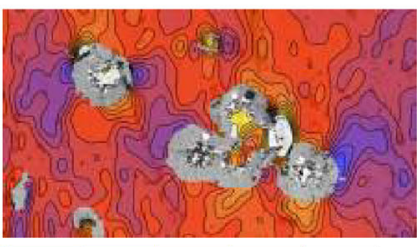

is

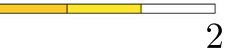

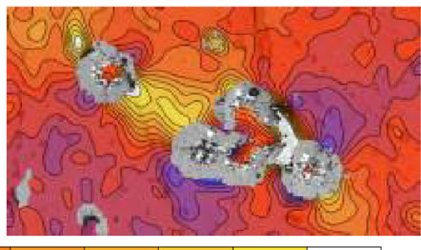

$\varepsilon_{x y}(\%)$ 
composites. $\varepsilon_{y}$ is maximal around the $0^{\circ}$ and $180^{\circ}$ of the single fiber while $\varepsilon_{x}$ exhibits strain concentrations around the single fiber (Fig. 11(b)), forming a cross shape similar to those observed for the PTFE / epoxy, PLA / epoxy and HCS / expoy specimens. $\varepsilon_{x y}$ 's distribution is also similar to that of single fibers, revealing a symmetrical shape with maximums at $45^{\circ}$ and $-135^{\circ}$ and minimums with opposite values at $45^{\circ}$ and $-135^{\circ}$ along the carbon fiber / epoxy interface. Figure 11(c) shows that interfacial debonding progresses on the edges of the small bundle, without affecting its core. Interfacial debonding also grows through the whole interface of the isolated fiber, completely debonding it. No debonding was visible around the fiber / matrix interface at the bundle's core, throughout the whole mechanical test.

\section{Out of plane displacements}

Figure 12 presents out of plane measurements for the CF / epoxy composite. The area presented is the same as that shown in Fig. 9, for $\bar{\sigma}=4.5 \mathrm{MPa}$ and $\bar{\varepsilon}=2.5 \%$, as shown in Figs. 10(c) and 11(c).

Following polishing, variations in out of plane measurements were found to be less than $2.5 \mu \mathrm{m}$ for the area presented in Fig. 9 (although the unloaded out of plane measurements were not presented in this work, they are available in the data package). Figure 12 shows that the surface observed, has now severely been deformed in the out of plane direction. The fibers within the large CF bundle, as detailed in Fig. 10(c), are now protruding out of the material at a height of about $12 \mu \mathrm{m}$ above the average height of the matrix far away from the bundle. The matrix has experienced compression along the out of plane direction $z$ due to the strain applied in the $y$ direction (Poisson's effect). Matrix areas entrapped within the fibers at the core of the bundle also appear to not have experienced any out of plane compression as their average height is about $10 \mu \mathrm{m}$. This delay in interfacial cracking between the bundle's core region and its edges is attributed to the strong matrix / fiber interface. The smaller fiber bundle detailed in Fig. 11(c) is also protruding out of the matrix, as shown in Fig. 12. The average height of the smaller bundle is about $8-10 \mu \mathrm{m}$ smaller than that of the larger bundle and also appears to continuously decrease from the bundle's center down to the matrix. Single fibers are also protruding out of the matrix, showing a clear discontinuity in out of plane measurements.

Figure 13a shows a cropped area around the single fiber shown in Fig. 9 for a stress level of $7.7 \mathrm{MPa}$ (e.g., Fig. 11(c)). The vertical and horizontal slide ruler shows that the in plane interfacial crack (black area around the fiber) is about $2 \mu \mathrm{m}$ at most. Figure 13(b) presents height measurements along the two profile lines (oriented along $x$ and $y$ ) shown in Fig. 13(a). $z=0$ was selected as the minimum out of plane value measured within Fig. 13(a) and is not visible in Fig. 13(b). The plateau at about $7 \mu \mathrm{m}$ of height corresponds to the CF's free surface. The plot shows that out of plane measurements sharply decrease along the $x$ and $y$ directions away from the fiber at first, then decrease progressively, as it can also be observed from Fig. 12. The out of plane interfacial crack is about $2 \mu \mathrm{m}$ at least, which is about the same size as the in plane interfacial crack. The debonding profile observed is similar to that observed for the HCS / epoxy specimen presented in Fig. 13(f).

\section{Discussion}

\section{Strong and Weak Bonding}

The PTFE, PLA and HCS single fibers studied in this work were selected based on their bonding strengths with epoxy, thus providing a case with no interface (PTFE), a case with a weak bonding (PLA), and a case with strong bonding (HCS). The interface strain localization strongly depended on the interface toughness (Fig. 6, 7(a) and (b)). The HCS /
Fig. 12 3D surface profile of the area presented in Fig. 9 for $\bar{\varepsilon}=$ $2.5 \%$ (see Figs. 10(c) and 11(c)). Height measurements are obtained using the laser confocal microscope [39]. A large bundle $(\approx 100$ fibers $)$, a smaller bundle $(\approx 5$ fibers $)$ and single fibers are visible, along with their out of plane displacement, shown as a contour plot

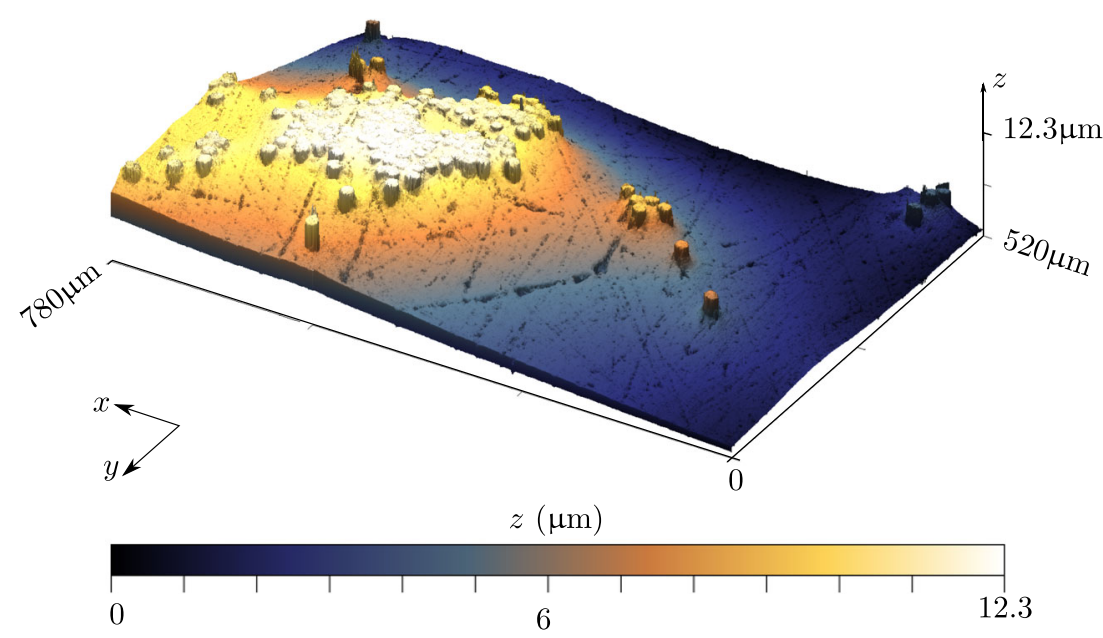


Fig. 13 (a) Cropped area around the single fiber shown in Fig. 9. Vertical and horizontal side rulers show that the in-plane interfacial crack appears to be about $2 \mu \mathrm{m}$ wide at most, it is shown with a red double arrow. (b) Height measurements along two profile lines shown in (a). The top of the CF is visible as a plateau at about $7 \mu \mathrm{m}$. Further away from the fiber (not visible in (b), shown in Fig. 12) the average matrix' height is $0 \mu \mathrm{m}$. The out of plane crack, distance between the matrix height and top of the $\mathrm{CF}$, is about $2 \mu \mathrm{m}$ wide

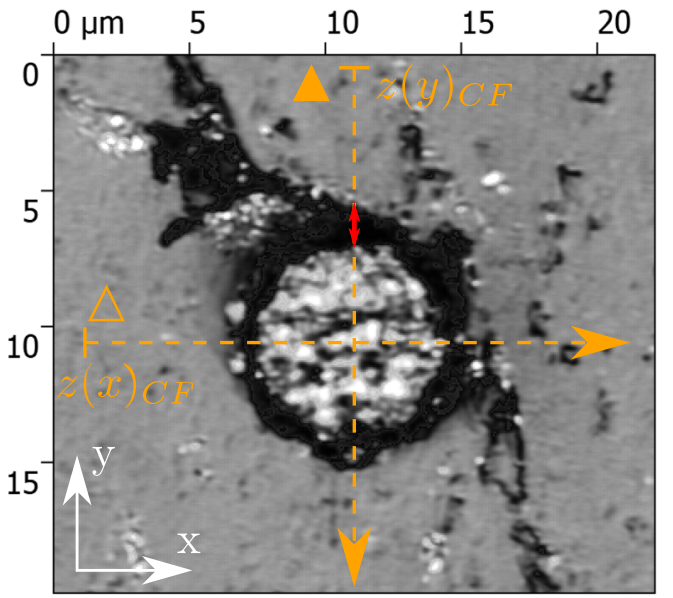

$\mathbf{a}$

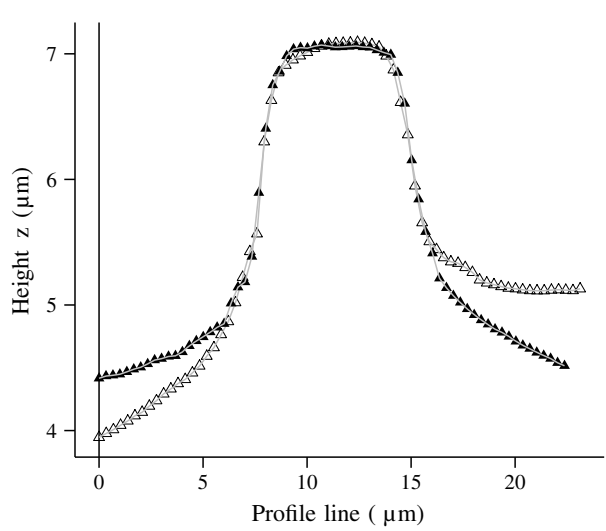

b epoxy composite was shown to behave differently than the PTFE / epoxy or PLA / epoxy when submitted to transverse loading (Section "Virtual extensometers"). Figure 6 shows that $\varepsilon_{\mathrm{y}}^{\mathrm{T}}$ increases at a faster than linear rate with respect to the applied global strain for the PTFE / epoxy composite and faster than that observed for the PLA / epoxy and HCS / epoxy composites. By opposition, $\varepsilon_{\mathrm{y}}^{\mathrm{T}}$ for the HCS / epoxy specimen shows that strong bonding hinders tangential opening (Mode II) around the interfacial areas at $90^{\circ}$ and $-90^{\circ}$. Figure 7 (b) also shows that the compressive strain applied by the matrix on the HCS fiber is negligible before $\bar{\varepsilon}=2 \%$. The matrix even appears to be pulling on the fiber, as $\varepsilon_{x}^{l}$ is be positive for the HCS / epoxy specimen at the beginning of the test. This behavior is unique to the HCS / epoxy specimen. Strong bonding could explain this behavior as it was also observed in a previous work involving $1 \mathrm{~mm}$ galvanized steel fibers in two different epoxies [40].

While the bundle of CFs is composed of clusters of single CFs, damage first occurs at the bundle's interface with the martix due to the coalescence of individual cracks located at the fiber / matrix interface of fibers at the bundle's periphery, while no particular damage was observed within its core. This early damage localization is analogous to that investigated in single fiber reinforced composites, when the fiber bundle is considered as a single fiber.

\section{Damage Initiation}

Damage initiation and growth of the interfacial crack for single fiber specimens happen differently for each configuration. Figure 7(a) shows that the interfacial crack immediately starts growing as $\varepsilon_{y}^{l}$ increases from the very beginning of the test, although the crack is not yet visible on the micrographs due to the optical setup resolution.
The interfacial crack around the PTFE fiber in Fig. 3(a) appears to only be present at the $0^{\circ}$ and $180^{\circ}$ areas. Later on during the test (Fig. 3(b)), no sign of interfacial debonding is visible around the $90^{\circ}$ and $-90^{\circ}$ interfacial areas. Both cracks $\left(0^{\circ}\right.$ and $\left.180^{\circ}\right)$ appear to be symmetrically growing at the same rate.

For the PLA / epoxy specimen, Fig. 4(a) shows that an interfacial crack starts growing around the $0^{\circ}$ interfacial area. For this specimen, interfacial decohesion is also visible all around the fiber as a discontinuity. Figure 4(b) also shows that the crack growth is not symmetric, as only the $0^{\circ}$ crack keeps growing. These two differences in behavior, with respect to those observed for the PTFE / epoxy specimen, are attributed to the weak bonding of PLA / epoxy. As out of plane and in plane debonding occurs, the interfacial crack visibly grows around the fiber, which is not the case for the PTFE / epoxy specimen as there is no bonding in that case. Interfacial bonding might arbitrarily be stronger in the $180^{\circ}$ interfacial area, promoting debonding around the $0^{\circ}$ interfacial area.

The interfacial crack of the HCS / epoxy specimen debonded between $135^{\circ}$ and $-90^{\circ}$ in the interface (Fig. 5(a)). The crack initiation and growth is not symmetric in this case. The interfacial area around $90^{\circ}$ appears to remain bonded throughout the whole test, favoring interfacial crack growth between $135^{\circ}$ and $45^{\circ}$ (Fig. 5(b) and (c)).

\section{Fiber Size Effect}

The size of the interfacial debonding crack might depend on the materials' mechanical properties, bonding strength and fiber diameter [11, 19]. A previous study involving two different epoxy matrices with a larger PTFE fiber showed that different matrices had an impact on displacement measurements. The PTFE fiber used in [40] had a diameter 
of $1000 \mu \mathrm{m}$ (called "macro" fiber), the matrix used was the same as the one used in this work.

Figure 14 presents $\varepsilon_{x}^{l}$ and $\varepsilon_{y}^{l}$ with respect to the global applied strain for the PTFE / epoxy specimen presented in this work and the PTFE / epoxy specimen presented in Tabiai et al. [40]. The virtual extensometers edges were situated about a subset away from the fiber's edge in each direction. The figure shows that both measurements are consistent and show the same behavior. Differences between $\varepsilon_{x}^{l}$ and $\varepsilon_{y}^{l}$ appear at global strains of $3 \%$. At this point, the interfacial debonding crack is already significant and might have a larger impact on $\varepsilon_{x}^{l}$ and $\varepsilon_{y}^{l}$, which might explain the small quantitative difference later on. Another possibility is that the placement of both virtual extensometers was not quite the same with respect to the fiber due to the subsets having different sizes in both works. The fiber size does not appear to significantly affect the interfacial debonding crack growth.

\section{Limitations of the Study}

Residual stresses inherent to the fabrication process must have developed for all FRC variants due to the different coefficient of thermal expansion (CTE) for fibers and matrix, as well as chemical shrinkage $[16,34]$. Although the

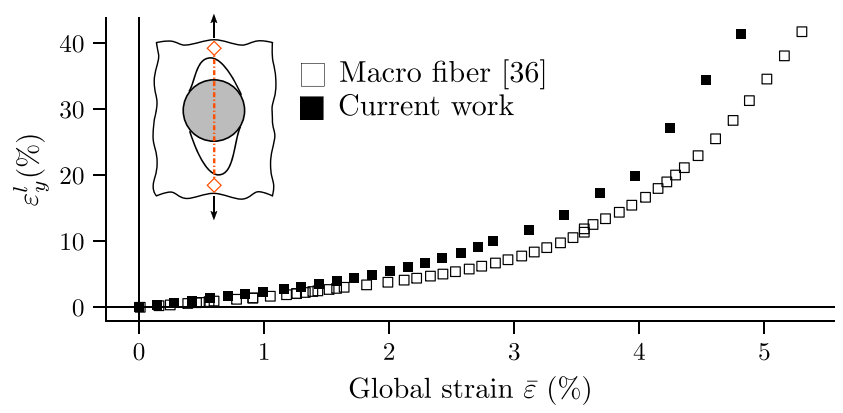

a

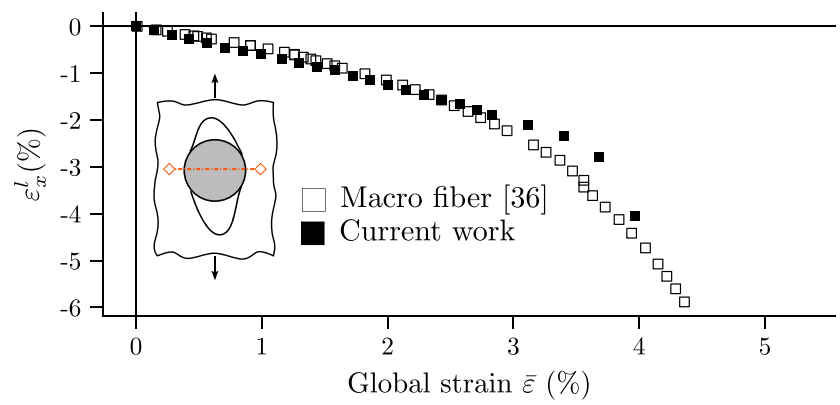

b

Fig. 14 Comparison of $\varepsilon_{x}^{l}$ and $\varepsilon_{y}^{l}$ for a PTFE macro fiber of $1000 \mu \mathrm{m}$ in diameter from [40] and results for the PTFE fiber $(711 \mu \mathrm{m})$ used in this work (presented in Fig. 7). Macro fiber results were obtained using a stereoscopic microscope equipped with two CCD cameras. DIC was carried out with VIC3D7 commercial software matrix selected cures at room temperature, it was possible to notice that the mold's temperature raised above room temperature during curing due to the exothermic nature of the polymerization reaction [41]. Temperature changes of the mold during curing were not measured during curing. In addition, during curing, cross-linking between linear chains induces a volume shrinkage [34, 42]. Residual stresses due to chemical shrinkage of the epoxy must also be present. The epoxy for this work was partially selected because it is possible to cure it at room temperature. In order to estimate these streses, rough and conservative simulations of the maximum residual stress around the fiber after curing showed that the residual stress is at most about 1.4 MPa for the Epoxy / HCS and around 0.6 MPa for the Epoxy / PTFE or Epoxy / PLA specimens.

For each single fiber specimen tested, the out of plane interfacial debonding is growing within the specimen around the fiber, along the $z$ direction. This mechanism, called tunneling [43], is directly linked to the cracks visible at the free surface [19]. The opening of the $0^{\circ}$ and $180^{\circ}$ cracks visible for the PTFE / epoxy and PLA / epoxy specimens (Figs. 3(c) and 4(c)) results from tunneling. Martyinuk et al. showed that free surface visible crack growth might be linearly linked to tunneling growth. Tunneling growth depends on the fiber / matrix bonding strength as debonding must continue along the fiber. The optical setup used in this work could not allow direct observation of this mechanism.

Interfacial debonding happens in plane and out of plane, simultaneously, for the CF / epoxy specimen, as shown in Fig. 12. Out of plane measurements, after interfacial debonding initiation, present a clear discontinuity between isolated carbon fibers and matrix. It can also be seen that out of plane interfacial debonding is particularly significant around the edges of the large CFs bundle where in plane interfacial debonding is also the most significant. Out of plane interfacial debonding is also present within the bundle's core but is less pronounced (inferior to $1 \mu \mathrm{m}$ ) than on the periphery. These results show that modeling this experiment should account for 3D effects as out of plane interfacial debonding was present during all tests and is as pronounced as in plane interfacial debonding. Height measurements for all specimens presented in this work, for each timestep, and a procedure to explore the dataset are provided in the additional package [44].

\section{Experimental Data Availability}

CSV files containing the displacement, height, strain, confidence $\mathcal{C}$ value for each pixel at each time-step, are made available as an additional dataset. The applied load and displacement for any image taken during the tests presented here is also made available. Each raw image 
is accompanied by a comma separated values file that provides the displacement, strain and confidence for each pixel visible on the raw image. A "readme PDF" file clarifying the format in which the data is structured is also provided. The calibration parameters obtained after calibrating each test are also included with the additional dataset. The images used for the calibration process are also provided in the additional dataset, meaning that this information can, for example, be used to spatially calibrate another DIC software to analyze the data differently, using different parameters [44].

The authors are making this data available to facilitate the exploration of the displacement and deformation fields around a fiber. This data can be used to better understand the damage mechanisms at the fiber level, or calibrate a damage model to obtain similar experimental results to those presented here, using simulation tools. These full field measurements of interfacial debonding can also be compared with results from different fiber / matrix couples.

\section{Conclusion}

The purpose of this study was to provide accurate full experimental displacement fields measurements of interfacial debonding and damage initiation in a fiber or bundle's vicinity with the goal of better understanding damage initiation and growth for fibers under transverse load. This work also provides out of plane measurements for fibers and a bundle of carbon fibers and connects the full displacement field measurements with out of plane displacements and measurements.

Damage in single fiber composites initiated at the fiber / matrix interface where an out of plane displacement difference between the fiber and matrix was the highest and where $\varepsilon_{y}$ was maximal $\left(0^{\circ}\right.$ and $\left.180^{\circ}\right)$. The interfacial debonding crack then grew around each single fiber. Interfacial debonding was shown to grow faster around PTFE and PLA fibers, which have the weakest bonding. Out of plane interfacial debonding is shown to play an important role in interfacial debonding as the size of the out of plane interfacial crack is larger than the in plane one for all specimens. Local measurements show that the strong bonding for the HCS fiber - epoxy bonding prevents tangential debonding around the $-90^{\circ}$ and $+90^{\circ}$ interface areas, slowing down the interfacial crack growth under Mode II. The bundle of CF showed how damage initiates and grows around a large bundle composed of about a hundred fibers and a small one containing only a few CF. Damage initiated at the edges of each bundle, which is also where $\varepsilon_{y}$ was maximal $\left(0^{\circ}\right.$ and $\left.180^{\circ}\right)$. Interfacial damage then grew around fibers on the edges without affecting the bundle's core. Out of plane interfacial debonding appears to be critical for single fibers, as the out of plane interfacial crack is larger than the in plane one. The larger bundle of CFs shows that carbon fibers concentration slows down out of plane interfacial crack growth. No visible in plane interfacial crack is visible at the bundle's core although less than $1 \mu \mathrm{m}$ out of plane cracks were measured within the core. A quantitative comparison of the displacement and strain fields around single fibers and a bundle of fibers is also provided here and shows that a bundle of carbon fibers can be assumed to behave, in overall, like a single fiber.

Extensive results for each specimen presented here are available online as an additional package. These results can be used to provide a better understanding of damage initiation and growth in a FRC. The geometry of specimens and reinforcements along with their elastic properties are provided. These results can also be used to quantitatively benchmark micromechanical damage models.

\section{References}

1. Talreja R, Veer Singh C (2012) Damage and failure of composite materials. Cambridge University Press, Cambridge University Press edition

2. Ageorges C, Friedrich K, Schüller T, Lauke B (1999) Singlefibre Broutman test: fibre-matrix interface transverse debonding. Compos A: Appl Sci Manuf 30:1423-1434

3. Pagano NJ (1998) On the micromechanical failure modes in a class of ideal brittle matrix composites. Part 1. Coated-fiber composites. Compos Part B: Eng 29:93-119

4. Romanowicz M (2010) Progressive failure analysis of unidirectional fiber-reinforced polymers with inhomogeneous interphase and randomly distributed fibers under transverse tensile loading. Compos A: Appl Sci Manuf 41:1829-1838

5. Hashin Z (1987) Analysis of damage in composite materials. In: Boehler JP (ed) Yielding, damage, and failure of anisotropic solids, vol 5. EGF Publication Edition

6. Hinton MJ, Kaddour AS, Soden PD (2004) Chapter 1.1 - the world-wide failure exercise: its origin, concept and content. In: Hinton MJ, Kaddour AS, Soden PD (eds) Failure criteria in fibre-reinforced-polymer composites. Elsevier, Oxford, pp 2-28

7. Kaddour A, Hinton M (2013) Maturity of $3 d$ failure criteria for fibre-reinforced composites: comparison between theories and experiments: Part B of WWFE-II, maturity of 3d failure criteria for fibre-reinforced composites: Comparison between theories and experiments: Part B of WWFE-II. J Compos Mater 47:925-966

8. Christensen RM (2014) The world wide failure exercise II examination of results - FailureCriteria.com

9. Shao J, Rudnicki J (2000) A microcrack-based continuous damage model for brittle geomaterials. Mech Mater 32:607-619

10. Nemat-Nasser S, Hori M (1993) Micromechanics: overall properties of heterogeneous materials. Amsterdam

11. Sørensen BF, Goutianos S (2014) Mixed mode cohesive law with interface dilatation. Mech Mater 70:76-93

12. Vernerey FJ, Kabiri M (2014) Adaptive concurrent multiscale model for fracture and crack propagation in heterogeneous media. Computer Methods in Applied Mechanics and Engineering

13. Sun Z, Zhao L, Chen L, Song Y (2013) Research on failure criterion of composite based on unified macro- and micromechanical model. Chin J Aeronaut 26:122-129 
14. Paris F, Correa E, Canas J (2003) Micromechanical view of failure of the matrix in fibrous composite materials. Compos Sci Technol 63:1041-1052

15. Wright P, Moffat A, Sinclair I, Spearing SM (2010) High resolution tomographic imaging and modelling of notch tip damage in a laminated composite. Compos Sci Technol 70:14441452

16. González C, LLorca J (2007) Mechanical behavior of unidirectional fiber-reinforced polymers under transverse compression: microscopic mechanisms and modeling. Compos Sci Technol 67:2795-2806

17. Burr A, Hild F, Leckie FA (1995) Micro-mechanics and continuum damage mechanics. Arch Appl Mech 65:437-456

18. Singh CV, Talreja R (2013) A synergistic damage mechanics approach to mechanical response of composite laminates with ply cracks. J Compos Mater 47:2475-2501

19. Martyniuk K, Sørensen BF, Modregger P, Lauridsen EM (2013) $3 \mathrm{~d}$ in situ observations of glass fibre/matrix interfacial debonding. Compos A: Appl Sci Manuf 55:63-73

20. Zhuang L, Pupurs A, Varna J, Talreja R, Ayadi Z (2018) Effects of inter-fiber spacing on fiber-matrix debond crack growth in unidirectional composites under transverse loading. Compos A: Appl Sci Manuf 109:463-471

21. Meurs PFM, Schrauwen BAG, Schreurs PJG, Peijs T (1998) Determination of the interfacial normal strength using single fibre model composites. Compos A: Appl Sci Manuf 29:1027-1034

22. Perrier A, Touchard F, Chocinski-Arnault L, Mellier D (2016) Mechanical behaviour analysis of the interface in single hemp yarn composites: DIC measurements and FEM calculations. Polym Test 52:1-8

23. Thomason JL, Yang L, Bryce D, Minty R (2016) An exploration of the relationship of chemical and physical parameters in the micromechanical characterisation of the apparent interfacial strength in glass fibre epoxy systems. IOP Conf Ser: Mater Sci Eng 139:012048

24. Canal L, González C, Molina-Aldareguía J, Segurado J, LLorca J. (2012) Application of digital image correlation at the microscale in fiber-reinforced composites. Compos A: Appl Sci Manuf 43:1630-1638

25. Mehdikhani M, Aravand M, Sabuncuoglu B, Callens MG, Lomov SV, Gorbatikh L (2016) Full-field strain measurements at the micro-scale in fiber-reinforced composites using digital image correlation. Compos Struct 140:192-201

26. Richefeu V, Chrysochoos A, Huon V, Monerie Y, Peyroux R, Wattrisse B (2012) Toward local identification of cohesive zone models using digital image correlation. Europ J Mech- A/Solids 34:38-51

27. Sakanashi Y, Gungor S, Forsey A, Bouchard P (2017) Measurement of creep deformation across welds in $316 \mathrm{~h}$ stainless steel using digital image correlation. Exp Mech 57:231-244

28. D20 Committee, Test Method for Tensile Properties of Plastics, Technical Report, ASTM International, (2010)
29. Petr Hlaváček JB (2012) Using of abrasive water jet for measurement of residual stress in railway wheels. Tehnicki Vjesnik 19:387-390

30. Awan IS, Xiaoqun W, Pengcheng H, Shanyi D (2012) Developing an approach to calculate carbon fiber surface energy using molecular simulation and its application to real carbon fibers. $\mathbf{J}$ Compos Mater 46:707-715

31. Guo S-Z, Gosselin F, Guerin N, Lanouette A-M, Heuzey M-C, Therriault D (2013) Solvent-cast three-dimensional printing of multifunctional microsystems. Small 9:4118-4122

32. Plummer J (2014) What makes epoxy resins good adhesives? Why do they bond so strongly to surfaces? Technical Report, Mereco Technologies, Londonderry, NH 03053

33. Pizzi A, Mittal KL (eds) (2003) Handbook of adhesive technology, 2nd edn. M. Dekker, New York. Rev and expanded edition

34. Parlevliet PP, Bersee HEN, Beukers A (2006) Residual stresses in thermoplastic composites-a study of the literature-part I: formation of residual stresses. Compos A: Appl Sci Manuf 37:1847-1857

35. Rong X, Keif M A study of PLA printability with flexography

36. Borodin AN, Sologubov AI, Chernenko LA, Zolkin PI, Aberyahimov HM, Grigoriev GA (1991) The influence of carbon fibre surface energy on their interfacial interaction with resins. In: Fridlyander IN, Kostikov VI (eds) MICC 90: Moscow international composites conference, 1990. Springer, Netherlands, pp 464-468

37. Sutton MA, Orteu J-J, Schreier HW (2009) Image correlation for shape, motion and deformation measurements: basic concepts, theory and applications. Springer

38. Correlated S (2010) Vic-3D help manual correlated solutions

39. Ins RB (2017) Olympic scientific solutions americas, laser confocal microscopy, challenging the limits of measuring surface roughness

40. Tabiai I, Delorme R, Therriault D, Levesque M (2018) In-situ full field measurements during inter-facial debonding in single fiber composite under transverse load. Experimental Mechanics

41. Prolongo SG, Gude MR, Ureña A (2010) The curing process of epoxy/amino-functionalized MWCNTs: calorimetry, molecular modelling, and electron microscopy. J Nanotechnol 2010:1-11

42. Abdel-Raheem NA, Halim SF, Al-Khoribi AH (2018) The effect of different curing conditions on hardness, thickness, and residual stress of carbon fiber reinforced epoxy composites. J Compos Mater 52:1959-1970

43. Ho S, Suo Z (1993) Tunneling cracks in constrained layers. J Appl Mech 60:890-894

44. Tabiai I, Texier D, Bocher P, Therriault D, Lévesque M (2018) Additional data for "In-situ full field out of plane displacement and strain measurements at the micro-scale in single reinforcement composites under transverse load. Type: dataset

Publisher's Note Springer Nature remains neutral with regard to jurisdictional claims in published maps and institutional affiliations. 


\section{Affiliations}

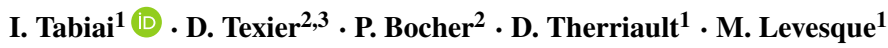

1 Laboratory for Multiscale Mechanics, Department of Mechanical Engineering, Polytechnique Montreal, 2900 boul.

Edouard-Montpetit, Montreal, QC, Canada

2 École de technologie superieure, 1100, rue Notre-Dame Ouest, Montreal, QC, Canada

3 Institut Clement Ader (ICA), Université de Toulouse, CNRS, INSA, UPS, Mines Albi, ISAE-SUPAERO, Campus Jarlard, 81013 Albi Cedex 09, France 